This is a self-archived version of an original article. This version may differ from the original in pagination and typographic details.

Author(s): Le Donne, Enrico; Leonardi, Gian Paolo; Monti, Roberto; Vittone, Davide

Title: Extremal polynomials in stratified groups

Year: 2018

Version: Accepted version (Final draft)

Copyright: (c) International Press of Boston, Inc.

Rights: In Copyright

Rights url: http://rightsstatements.org/page//nC/1.0/?language=en

Please cite the original version:

Le Donne, E., Leonardi, G. P., Monti, R., \& Vittone, D. (2018). Extremal polynomials in stratified groups. Communications in Analysis and Geometry, 26(4), 723-757.

https://doi.org/10.4310/CAG.2018.v26.n4.a3 


\title{
Extremal polynomials in stratified groups
}

\author{
Enrico Le Donne, Gian Paolo Leonardi, \\ Roberto Monti, and Davide Vittone
}

\begin{abstract}
We introduce a family of extremal polynomials associated with the prolongation of a stratified nilpotent Lie algebra. These polynomials are related to a new algebraic characterization of abnormal sub-Riemannian extremals in stratified nilpotent Lie groups. They satisfy a set of remarkable structure relations that are used to integrate the adjoint equations, in both normal and abnormal case.
\end{abstract}

$\begin{array}{lll}1 & \text { Introduction } & 723\end{array}$

\begin{tabular}{|lll}
\hline 2 & Structure formulas for extremal polynomials & $\mathbf{7 2 8}$
\end{tabular}

$\begin{array}{lll}3 & \text { Characterization of abnormal extremals } & 742\end{array}$

\begin{tabular}{|lll}
\hline 4 & The abnormal set in the free group of rank 2 and step 4 & 746
\end{tabular}

$\begin{array}{lll}5 & \text { Spiral-like Goh extremals } & 752\end{array}$

\begin{tabular}{ll}
\hline Acknowledgments & 754
\end{tabular}

\begin{tabular}{ll}
\hline References & 754
\end{tabular}

\section{Introduction}

Let $G$ be a stratified nilpotent Lie group of dimension $n$ and rank $r$. The Lie algebra $\mathfrak{g}=\operatorname{Lie}(G)$ has the stratification $\mathfrak{g}=\mathfrak{g}_{1} \oplus \cdots \oplus \mathfrak{g}_{s}$, where $s$ is the step of the algebra, $\mathfrak{g}_{i}=\left[\mathfrak{g}_{i-1}, \mathfrak{g}_{1}\right]$ for $i=2, \ldots, s+1$, and $\mathfrak{g}_{i}=\{0\}$ for $i>s$. The rank of $\mathfrak{g}$ is $r=\operatorname{dim}\left(\mathfrak{g}_{1}\right)$. Let $X_{1}, \ldots, X_{n}$ be a basis of $\mathfrak{g}$ adapted to the stratification. When it is connected and simply connected, we can identify the group $G$ with $\mathbb{R}^{n}$ via exponential coordinates of the second type

Key words and phrases: abnormal extremals, extremal polynomials, Carnot groups, sub-Riemannian geometry. 
induced by the basis $X_{1}, \ldots, X_{n}$, and we identify $\mathfrak{g}$ with the corresponding Lie algebra of left-invariant vector fields in $\mathbb{R}^{n}$.

Let $\operatorname{Prol}(\mathfrak{g})=\bigoplus_{k \leq s} \mathfrak{g}_{k}$ be the Tanaka prolongation of $\mathfrak{g}$, see [19]. Even though this is not strictly needed in our argument, an explicit construction is briefly recalled in Section 2. We extend $X_{1}, \ldots, X_{n}$ to a basis $\left\{X_{j}\right\}_{j \leq n}$ of the prolongation and to each $j \leq n$ we assign the degree $d(j)=k$ if and only if $X_{j} \in \mathfrak{g}_{k}$. Then we assume that the basis is adapted to the graduation: this means that $i<j$ implies $d(i) \leq d(j)$. When the prolongation is finite dimensional, the index $j$ ranges in a finite set, $m \leq j \leq n$ for some $m \in \mathbb{Z}$. With abuse of notation, we denote the basis $\left\{X_{j}\right\}_{m \leq j \leq n}$ by $\left\{X_{j}\right\}_{j \leq n}$, as in the infinite dimensional case. Let $c_{i j}^{k} \in \mathbb{R}$ be the structure constants of $\operatorname{Prol}(\mathfrak{g})$ associated with the basis $\left\{X_{j}\right\}_{j \leq n}$. Namely, for all $i, j \in \mathbb{Z}$ with $i, j \leq n$ we have

$$
\left[X_{i}, X_{j}\right]=\sum_{k \leq n} c_{i j}^{k} X_{k}
$$

The sum is always finite, because each stratum $\mathfrak{g}_{k}$ of $\operatorname{Prol}(\mathfrak{g})$ is finite dimensional and $c_{i j}^{k}=0$ if $d(k) \neq d(i)+d(j)$.

In this paper, we introduce a family of extremal polynomials $P_{j}^{v}(x), j \leq n$ and $x \in \mathbb{R}^{n}$, associated with the basis $\left\{X_{j}\right\}_{j \leq n}$ of $\operatorname{Prol}(\mathfrak{g})$. They depend linearly on a parameter $v \in \mathbb{R}^{n}$, see Definition 2.1. Extremal polynomials satisfy the following structure formulas.

Theorem 1.1. For any $v \in \mathbb{R}^{n}, i=1, \ldots, n$, and $j \in \mathbb{Z}$ with $j \leq n$ there holds

$$
X_{i} P_{j}^{v}(x)=\sum_{k \leq n} c_{i j}^{k} P_{k}^{v}(x), \quad x \in \mathbb{R}^{n} .
$$

Modulo the value at $x=0$, extremal polynomials are uniquely determined by the family of identities 1.2 . These structure formulas are the core of the paper and of our main technical result, Theorem 2.2. They first appeared in [9], but only in the case of free groups. In [9], the formulas were obtained a posteriori, as a consequence of certain algebraic identities, however only for $j=1, \ldots, n$ with no reference to the prolongation and only for the coordinates related to a Hall basis.

It is possible to give a coordinate-free definition of extremal polynomials, see [11. However, for the applications considered in Sections 4 and 5, as well as for the applications given in [9], it was crucial to possess explicit formulae for the polynomials (Definition 2.1). 
Our interest in extremal polynomials origins in the regularity problem of sub-Riemannian length minimizing curves, one of the main open problems in the field (see [13, [12], [2], [15], [20], [14], [10], 6], [17]). Let $M$ be a differentiable manifold and $\mathcal{D} \subset T M$ a bracket generating distribution. A Lipschitz curve $\gamma:[0,1] \rightarrow M$ is horizontal if $\dot{\gamma}(t) \in \mathcal{D}(\gamma(t))$ for a.e. $t \in[0,1]$. Fixing a quadratic form on $\mathcal{D}$, one can define the length of horizontal curves. Length minimizing curves may be either normal extremals or abnormal extremals: while normal extremals are always smooth, abnormal ones are a priori only Lipschitz continuous. Abnormal extremals depend only on the structure $(M, \mathcal{D})$ : they are precisely the singular points of the end-point mapping.

In Section 3, we use extremal polynomials and Theorem 1.1 to give an algebraic characterization of abnormal extremals in stratified nilpotent Lie groups (Carnot groups). This is of special interest because, by Mitchell's theorem, Carnot groups are the infinitesimal model of equiregular subRiemannian structures.

Let $\vartheta_{1}, \ldots, \vartheta_{n}$ be a basis of 1 -forms of $\mathfrak{g}^{*}$, the dual of $\mathfrak{g}$. A curve $\lambda \in$ $\operatorname{Lip}\left([0,1] ; \mathfrak{g}^{*}\right)$ is given by coordinates $\lambda_{1}, \ldots, \lambda_{n} \in \operatorname{Lip}([0,1])$ such that $\lambda=$ $\lambda_{1} \vartheta_{1}+\cdots+\lambda_{n} \vartheta_{n}$. If $\lambda$ is the dual curve of a normal or abnormal extremal $\gamma:[0,1] \rightarrow G=\mathbb{R}^{n}$, then

$$
\dot{\lambda}_{i}=-\sum_{k=1}^{n} \sum_{j=1}^{r} c_{i j}^{k} \dot{\gamma}_{j} \lambda_{k} \quad \text { a.e. on }[0,1], \quad i=1, \ldots, n \text {. }
$$

See Section 3 and Theorem 3.1 for more details. In Theorem 3.4, we use the structure formulas 1.2 to integrate the system of adjoint equations (1.3). The solutions are

$$
\lambda_{i}(t)=P_{i}^{v}(\gamma(t)), \quad i=1, \ldots, n,
$$

where $v \in \mathbb{R}^{n}$ is such that $v_{i}=\lambda_{i}(0)$. Thus, we can prove the following theorem (see Theorem 3.8 for the complete statement).

Theorem 1.2. Let $G=\mathbb{R}^{n}$ be a stratified nilpotent Lie group and let $\gamma$ : $[0,1] \rightarrow G$ be a horizontal curve with $\gamma(0)=0$. Then, the following statements are equivalent:

(A) The curve $\gamma$ is an abnormal extremal.

(B) There exist $v \in \mathbb{R}^{n}, v \neq 0$, such that $P_{i}^{v}(\gamma(t))=0$ for all $t \in[0,1]$ and for all $i \leq r$. 
Abnormal extremals are precisely the horizontal curves lying inside algebraic varieties defined via extremal polynomials. This result extends 9 , Theorem 1.1] because it applies to nonfree Carnot groups. It also improves that result because abnormal curves are shown to be in algebraic varieties smaller than those considered in [9]. Notice that in (B) also indexes $i \leq 0$ related to the Tanaka prolongation are involved.

Extremal polynomials can be also used to find explicit prime integrals of the Hamiltonian system associated with the group. In fact, a normal extremal $(\gamma, \lambda)$, where $\gamma$ is a curve in the group and $\lambda$ is the dual curve in the cotangent bundle, solves the system of equations

$$
\dot{\gamma}=\frac{\partial H}{\partial \lambda}(\gamma, \lambda), \quad \dot{\lambda}=-\frac{\partial H}{\partial \gamma}(\gamma, \lambda)
$$

where $H$ is the natural Hamiltonian function. Thanks to the Lie group structure, by Noether's theorem the Hamiltonian system (1.5) has $n$ prime integrals, where $n$ is the dimension of the group. In the coordinates introduced above, the equation $\dot{\lambda}=-\partial H / \partial \gamma$ becomes precisely equation (1.3). Thus, the solutions (1.4) provide $n$ prime integrals for the system (1.5).

The role of Tanaka prolongation in our theory is rather subtle. In a first attempt, we searched for an integration algorithm inverting the differentiation process that now is established by the structure formulas $(1.2)$. Part of statement (B) in Theorem 1.2 is that an abnormal extremal $\gamma$ satisfies $P_{i}^{v}(\gamma)=0$ for some $v \neq 0$ and for all $i=1, \ldots, r$. Thus, for any $j=1, \ldots, n$ also the functions

$$
B_{i j}^{v}(t)=\int_{0}^{t} P_{i}^{v}(\gamma(s)) \dot{\gamma}_{j}(s) d s, \quad t \in[0,1]
$$

vanish. Now, if the functions $B_{i j}^{v}(t)$ are polynomials of the coordinates $\gamma_{1}(t)$, $\ldots, \gamma_{n}(t)$, then we get new polynomials vanishing along the curve $\gamma$. However, this may or may not be the case and we were not able to understand this phenomenon in general. In Section 4, we show an example where the integration process (1.6) succeeds and, as a matter of fact, provides precisely the extremal polynomials associated with the layer $\mathfrak{g}_{0}$ of the Tanaka prolongation, the polynomials 4.49.

Tanaka prolongation has the following role in our argument. If $P_{j}^{v}$ is an extremal polynomial associated with the layer $\ell \leq 0$ of the prolongation (i.e., $d(j)=\ell$ ) and $\gamma$ is a horizontal curve, then by the structure formulas 
(1.2) we have

$$
\frac{d}{d t} P_{j}^{v}(\gamma(t))=\sum_{i=1}^{r} \sum_{k \leq n} c_{i j}^{k} \dot{\gamma}_{i}(t) P_{k}^{v}(\gamma(t)), \quad t \in[0,1],
$$

where the sum in $k$ ranges over indices with $d(k) \geq \ell+1$, because of the stratification. If the right-hand side vanishes, then also the left-hand side does and inductively, starting from the layer with $\ell=1$ descending, we can prove that abnormal extremals are in the zero set of all extremal polynomials given by the layers with $\ell \leq 0$ of the prolongation (see Theorem 3.8).

In the final part of the paper, we show two applications of the theory.

In Section 4, we develop a technique to construct a nontrivial algebraic set containing all abnormal extremals passing through one point. This is related to the problem of estimating the size of the set of regular values of the end-point mapping (see [2] and [13, Section 10.2]). In sub-Riemannian manifolds with a distribution of corank 1, the image of the set of length minimizing abnormal extremals starting from one point has zero Lebesgue measure; on the other hand, independently from corank, the image of strictly abnormal length minimizing extremals has empty interior (see Corollary 3 in [18] and [1]).

Our technique seems to work when the prolongation is sufficiently large. In this case, for each abnormal curve $\gamma$ there is at least one parameter $v \in \mathbb{R}^{n}$ and many indexes $j$ such that $P_{j}^{v}(\gamma)=0$. It is then possible to find a polynomial $Q$ independent of $v$ such that $Q(\gamma)=0$ for any abnormal curve $\gamma$ passing through one fixed point. We describe the technique in detail in the case of the free nilpotent Lie group of rank 2 and step 4 . However, it can be implemented in many other examples and it is likely to work in any nonrigid group.

Finally, in Section 5 we construct a 64-dimensional Lie group possessing a spiral-like Goh extremal whose tangents at the singular point are all lines. This example points out a limitation of the shortening technique introduced in [12] and developed in [14, [16], and [6]. It is also interesting in relation to the examples of nonrectifiable spiral-like rigid paths studied in [23] and [22], whereas our spiral-like extremal has finite length. As Example 6.4 in [9], it also shows that from the first and second order conditions (Pontryagin Maximum Principle and Goh condition) it is not possible to deduce any further regularity for abnormal minimizers beyond the Lipschitz regularity. 


\section{Structure formulas for extremal polynomials}

Let $X_{1}, \ldots, X_{n}$ be a basis of $\mathfrak{g}=\operatorname{Lie}(G)$ adapted to the stratification. We identify the group $G$ with $\mathbb{R}^{n}$ via exponential coordinates of the second type induced by the basis $X_{1}, \ldots, X_{n}$. Namely, for any $x=\left(x_{1}, \ldots, x_{n}\right) \in \mathbb{R}^{n}$ we have

$$
x=\mathrm{e}^{x_{1} X_{1}} \circ \cdots \circ \mathrm{e}^{x_{n} X_{n}}(0)=\exp \left(x_{n} X_{n}\right) \cdots \exp \left(x_{1} X_{1}\right) .
$$

Above, exp : $\mathfrak{g} \rightarrow G$ is the exponential mapping, $\cdot$ is the group law in $G=\mathbb{R}^{n}$, and $\gamma(t)=\mathrm{e}^{t X}(x)=x \exp (t X), t \in \mathbb{R}$, is the solution of the Cauchy Problem

$$
\left\{\begin{array}{l}
\dot{\gamma}=X(\gamma) \\
\gamma(0)=x
\end{array}\right.
$$

In simply connected nilpotent groups, the exponential mapping is a global diffeomorphism. After the identification (2.7), the Lie algebra $\mathfrak{g}$ is isomorphic to a Lie algebra of vector fields in $\mathbb{R}^{n}$ that are left invariant with respect to the group law $\cdot$

We recall the construction of the Tanaka prolongation. First, we define the vector space of all strata-preserving derivations of $\mathfrak{g}$ :

$$
\mathfrak{g}_{0}=\left\{\phi \in \bigoplus_{i=1}^{s} \mathfrak{g}_{i} \otimes \mathfrak{g}_{i}^{*}: \phi([X, Y])=[\phi(X), Y]+[X, \phi(Y)], X, Y \in \mathfrak{g}\right\} .
$$

Recall that $\mathfrak{g}_{i} \otimes \mathfrak{g}_{i}^{*}$ is canonically isomorphic to $\operatorname{End}\left(\mathfrak{g}_{i}\right)$. The direct sum vector space $\bigoplus_{i=0}^{s} \mathfrak{g}_{i}$ is a graded Lie algebra with the bracket

$$
[\phi, X]=-[X, \phi]=\phi(X), \quad \text { for all } \phi \in \mathfrak{g}_{0} \text { and } X \in \mathfrak{g},
$$

and with the natural bracket on $\mathfrak{g}$ and $\mathfrak{g}_{0}$.

By induction, assume that we have a vector space $\bigoplus_{i=1-k}^{s} \mathfrak{g}_{i}$ for some $k \geq 1$ and assume that the bracket $[\phi, X]=\phi(X)$ is already defined for all $\phi \in \bigoplus_{i=1-k}^{s} \mathfrak{g}_{i}$ and $X \in \mathfrak{g}$. Then we define the vector space of all derivations $\phi: \mathfrak{g} \rightarrow \mathfrak{g}_{1-k} \oplus \cdots \oplus \mathfrak{g}_{s-k}$ such that $\phi\left(\mathfrak{g}_{i}\right) \subset \mathfrak{g}_{i-k}$ :

$\mathfrak{g}_{-k}=\left\{\phi \in \bigoplus_{i=1}^{s} \mathfrak{g}_{i-k} \otimes \mathfrak{g}_{i}^{*}: \phi([X, Y])=[\phi(X), Y]+[X, \phi(Y)], X, Y \in \mathfrak{g}\right\}$. 
Recall that $\mathfrak{g}_{i-k} \otimes \mathfrak{g}_{i}^{*}$ is isomorphic to $\operatorname{Hom}\left(\mathfrak{g}_{i} ; \mathfrak{g}_{i-k}\right)$. As above, the direct sum vector space $\bigoplus_{i=-k}^{s} \mathfrak{g}_{i}$ is a graded Lie algebra with the bracket

$$
[\phi, X]=-[X, \phi]=\phi(X), \quad \text { for all } \phi \in \mathfrak{g}_{-k} \text { and } X \in \mathfrak{g},
$$

with the natural bracket on $\mathfrak{g}$, and with the bracket $[\phi, \psi]$ for $\phi, \psi \in \mathfrak{g}_{-k} \oplus$ $\cdots \oplus \mathfrak{g}_{0}$ defined by

$$
[\phi, \psi](X)=[[\phi, X], \psi]+[\phi,[\psi, X]], \quad X \in \mathfrak{g} .
$$

This inductive construction may or may not end after a finite number of steps, i.e., we have either $\mathfrak{g}_{-k}=\{0\}$ for some $k \geq 1$ or $\mathfrak{g}_{-k} \neq\{0\}$ for all $k \geq 1$. In both cases, we let

$$
\operatorname{Prol}(\mathfrak{g})=\bigoplus_{k \leq s} \mathfrak{g}_{k}
$$

$\operatorname{Prol}(\mathfrak{g})$ is a graded Lie algebra, called Tanaka prolongation of $\mathfrak{g}$. Namely, we have

$$
\left[\mathfrak{g}_{i}, \mathfrak{g}_{j}\right] \subset \mathfrak{g}_{i+j}, \quad \text { for all } i, j \in \mathbb{Z} \text { with } i, j, i+j \leq s .
$$

This is the unique property that we need in the proof of the structure theorem of extremal polynomials, Theorem 2.2. In fact, we need (2.9) only for $j=1, \ldots, s$. We do not specifically need the Tanaka prolongation but only a graded Lie algebra extending $\mathfrak{g}$ and satisfying (2.8) and (2.9). Among all such extensions $\operatorname{Prol}(\mathfrak{g})$ is the largest one.

In general, the explicit computation of $\operatorname{Prol}(\mathfrak{g})$ is difficult. When $\mathfrak{g}$ is a free nilpotent Lie algebra of step $s \geq 3$, then we have $\operatorname{Prol}(\mathfrak{g})=\mathfrak{g}_{0} \oplus \mathfrak{g}$, with the exceptional case of the step 3 and rank 2 free Lie algebra (see [21]). let

Let $\left\{X_{j}\right\}_{j \leq n}$ be a basis of $\operatorname{Prol}(\mathfrak{g})$. For integers $j_{0}, j_{1}, j_{2}, \ldots, j_{k} \leq n$, we

$$
\left[X_{j_{0}}, X_{j_{1}}, X_{j_{2}}, X_{j_{3}}, \ldots, X_{j_{k}}\right]=\left[\cdots\left[\left[\left[X_{j_{0}}, X_{j_{1}}\right], X_{j_{2}}\right], X_{j_{3}}\right], \ldots, X_{j_{k}}\right] .
$$

Then, for $\alpha=\left(\alpha_{1}, \ldots, \alpha_{n}\right) \in \mathcal{I}=\mathbb{N}^{n}=\{0,1,2, \ldots\}^{n}$, we define the iterated commutator of $X_{1}, \ldots, X_{n}$

$$
\left[\cdot, X_{\alpha}\right]=[\cdot, \underbrace{X_{1}, \ldots, X_{1}}_{\alpha_{1} \text { times }}, \underbrace{X_{2}, \ldots, X_{2}}_{\alpha_{2} \text { times }}, \ldots, \underbrace{X_{n}, \ldots, X_{n}}_{\alpha_{n} \text { times }}] .
$$

Here, only elements $X_{1}, \ldots, X_{n}$ of the basis of $\mathfrak{g}$ are involved. We agree that $\left[\cdot, X_{(0, \ldots, 0)}\right]=\mathrm{Id}$. The generalized structure constants $c_{i \alpha}^{k} \in \mathbb{R}$, with $\alpha \in \mathcal{I}=$ 
$\mathbb{N}^{n}$ and $i, k \in \mathbb{Z}$ such that $i, k \leq n$, are defined via the relation

$$
\left[X_{i}, X_{\alpha}\right]=\sum_{k \leq n} c_{i \alpha}^{k} X_{k}
$$

The above sum is always finite. In fact, letting $d(\alpha)=\sum_{j=1}^{n} \alpha_{j} d(j)$, we have $c_{i \alpha}^{k}=0$ if $d(k) \neq d(i)+d(\alpha)$.

For $\alpha \in \mathcal{I}$ and $i \in \mathbb{Z}$ with $i \leq n$, we define the linear mapping $\phi_{i \alpha} \in$ $\operatorname{Hom}\left(\mathbb{R}^{n} ; \mathbb{R}\right)$

$$
\phi_{i \alpha}(v)=\frac{(-1)^{|\alpha|}}{\alpha !} \sum_{k \leq n} c_{i \alpha}^{k} v_{k}, \quad v=\left(v_{1}, \ldots, v_{n}\right) \in \mathbb{R}^{n} .
$$

Above, we let $\alpha !=\alpha_{1} ! \cdots \alpha_{n} !$. Moreover, we agree that $v_{k}=0$ for $k \leq 0$.

Definition 2.1. For each $i \in \mathbb{Z}$ with $i \leq n$ and $v \in \mathbb{R}^{n}$, we call the polynomial $P_{i}^{v}: \mathbb{R}^{n} \rightarrow \mathbb{R}$

$$
P_{i}^{v}(x)=\sum_{\alpha \in \mathcal{I}} \phi_{i \alpha}(v) x^{\alpha}, \quad x \in \mathbb{R}^{n}
$$

extremal polynomial of the Lie algebra $\operatorname{Prol}(\mathfrak{g})$ with respect to the basis $\left\{X_{j}\right\}_{j \leq n}$.

For any finite set of multi-indexes $\mathcal{A} \subset \mathcal{I}$, consider the polynomial

$$
P(x)=\sum_{\alpha \in \mathcal{A}} c_{\alpha} x^{\alpha}
$$

where $c_{\alpha} \in \mathbb{R}$ for all $\alpha \in \mathcal{A}$. The homogeneous degree of the polynomial is $d(P)=\max \left\{d(\alpha): \alpha \in \mathcal{A}\right.$ such that $\left.c_{\alpha} \neq 0\right\}$. We say that the polynomial is homogeneous of degree $k \geq 0$ if $d(\alpha)=k$ for all $\alpha \in \mathcal{A}$ such that $c_{\alpha} \neq 0$.

Extremal polynomials $P_{i}^{v}$ are indeed polynomials. In fact, if $d(i)+d(\alpha) \neq$ $d(k)$ then we have $c_{i \alpha}^{k}=0$. Moreover, we have $d(k) \leq d(n)=s$ and therefore the sum in 2.14) ranges over the $\alpha \in \mathcal{I}$ such that $d(\alpha) \leq s-d(i)$. So we have $d\left(P_{i}^{v}\right) \leq s-d(i)$.

The following theorem is the main result of the paper. 
Theorem 2.2. For any $v \in \mathbb{R}^{n}, i=1, \ldots, n$, and $j \in \mathbb{Z}$ with $j \leq n$ there holds

$$
X_{i} P_{j}^{v}=\sum_{k \leq n} c_{i j}^{k} P_{k}^{v}
$$

Moreover, the polynomials $\left\{P_{j}^{v}\right\}_{j \leq n}$ are uniquely determined by 2.15 for $i=1, \ldots, r$ and $P_{j}^{v}(0)=v_{j}$ for $j \leq n$.

The identity $P_{j}^{v}(0)=v_{j}$ is proved in 2.36 below. The uniqueness follows from this observation: if $f$ is a smooth function on $G=\mathbb{R}^{n}$ such that $X_{i} f=$ 0 for all $i=1, \ldots, r$, then $f$ is constant. Now, let $\left\{Q_{j}^{v}\right\}_{j \leq n}$ be a family of polynomials satisfying 2.15 for $i=1, \ldots, r$, and $Q_{j}^{v}(0)=v_{j}$ for $j \leq n$. Then, for $d(j)=s$ we have $X_{i} P_{j}^{v}=0=X_{i} Q_{j}^{v}$ and thus $P_{j}^{v}-Q_{j}^{v}=P_{j}^{v}(0)-$ $Q_{j}^{v}(0)=0$. Assume by induction that $P_{j}^{v}=Q_{j}^{v}$ for $d(j) \geq \ell+1$ and take $j \leq n$ such that $d(j)=\ell$. From 2.15, we have

$$
X_{i} P_{j}^{v}=\sum_{k \leq n} c_{i j}^{k} P_{k}^{v}=\sum_{k \leq n} c_{i j}^{k} Q_{k}^{v}=X_{i} Q_{j}^{v}, \quad i=1, \ldots, r
$$

and thus $P_{j}^{v}-Q_{j}^{v}=P_{j}^{v}(0)-Q_{j}^{v}(0)=0$.

Before starting the proof of (2.15), we need three preliminary lemmas. For $i=1, \ldots, n$, let $G_{i} \subset G=\mathbb{R}^{n}$ be the subgroup of $G$

$$
\begin{aligned}
G_{i} & =\left\{x \in G=\mathbb{R}^{n}: x_{1}=\cdots=x_{i-1}=0\right\} \\
& =\left\{\mathrm{e}^{x_{i} X_{i}} \circ \cdots \circ \mathrm{e}^{x_{n} X_{n}}(0) \in G=\mathbb{R}^{n}: x_{i}, \ldots, x_{n} \in \mathbb{R}\right\} .
\end{aligned}
$$

We clearly have $G_{1}=G$. The following lemma is well-known and we omit the proof.

Lemma 2.3. Let $X_{1}, \ldots, X_{n}$ be vector fields in $\mathbb{R}^{n}$ satisfying 2.7). Then:

(i) For all $i=1, \ldots, n$ and for all $x \in G_{i}$ we have $X_{i}(x)=\partial / \partial x_{i}$.

(ii) There exist functions $f_{i \ell}: \mathbb{R}^{n} \rightarrow \mathbb{R}, i, \ell=1, \ldots, n$, such that

$$
X_{i}(x)=\frac{\partial}{\partial x_{i}}+\sum_{\ell: d(\ell)>d(i)} f_{i \ell}(x) \frac{\partial}{\partial x_{\ell}}, \quad x \in G=\mathbb{R}^{n} .
$$

In the following two lemmas, we prove formula 2.15 in two simplified situations that will serve as base for a long induction argument. 
Lemma 2.4. For any $i=1, \ldots, n, j \in \mathbb{Z}$ with $j \leq n$, and $v \in \mathbb{R}^{n}$ there holds

$$
X_{i} P_{j}^{v}=\sum_{k \leq n} c_{i j}^{k} P_{k}^{v} \quad \text { on } G_{i}
$$

Proof. For $x \in G_{i}$, we have

$$
P_{j}^{v}(x)=\sum_{\ell \leq n} \sum_{\alpha \in \mathcal{I}_{i}} c_{\alpha} c_{j \alpha}^{\ell} v_{\ell} x^{\alpha}
$$

where we let $\mathcal{I}_{i}=\left\{\alpha \in \mathcal{I}: \alpha_{1}=\cdots=\alpha_{i-1}=0\right\}$ and

$$
c_{\alpha}=\frac{(-1)^{|\alpha|}}{\alpha !} \text {. }
$$

By (i) in Lemma 2.3, on $G_{i}$ we have $X_{i}=\partial / \partial x_{i}$. Then, differentiating 2.17) we obtain

$$
X_{i} P_{j}^{v}(x)=-\sum_{\ell \leq n} \sum_{\beta \in \mathcal{I}_{i}} c_{\beta} c_{j, \beta+\mathrm{e}_{i}}^{\ell} v_{\ell} x^{\beta}, \quad x \in G_{i} .
$$

On the other hand, for $x \in G_{i}$ and $v \in \mathbb{R}^{n}$ we have

$$
\sum_{k \leq n} c_{i j}^{k} P_{k}^{v}(x)=\sum_{\ell, k \leq n} \sum_{\beta \in \mathcal{I}_{i}} c_{\beta} c_{i j}^{k} c_{k \beta}^{\ell} v_{\ell} x^{\beta}
$$

By (2.18) and 2.19), proving the claim 2.16) is equivalent to show that for all $\ell \leq n$ and for all $\beta \in \mathcal{I}_{i}$ we have

$$
-c_{j, \beta+\mathrm{e}_{i}}^{\ell}=\sum_{k \leq n} c_{i j}^{k} c_{k \beta}^{\ell}
$$

As $\beta \in \mathcal{I}_{i}$, there holds $-\left[X_{j}, X_{\beta+\mathrm{e}_{i}}\right]=\left[\left[X_{i}, X_{j}\right], X_{\beta}\right]$, and thus, by 2.12 and (1.1), we obtain

$$
\begin{aligned}
-\sum_{\ell \leq n} c_{j, \beta+\mathrm{e}_{i}}^{\ell} X_{\ell} & =-\left[X_{j}, X_{\beta+\mathrm{e}_{i}}\right]=\left[\left[X_{i}, X_{j}\right], X_{\beta}\right] \\
& =\sum_{k \leq n} c_{i j}^{k}\left[X_{k}, X_{\beta}\right]=\sum_{\ell, k \leq n} c_{i j}^{k} c_{k \beta}^{\ell} X_{\ell} .
\end{aligned}
$$

Since $\left\{X_{\ell}\right\}_{\ell \leq n}$ is a basis, this proves 2.20 . 
Lemma 2.5. For all $j \in \mathbb{Z}$ with $j \leq n, i=1, \ldots, n$ such that $d(i)=s$, and $v \in \mathbb{R}^{n}$ there holds

$$
X_{i} P_{j}^{v}=\sum_{k \leq n} c_{i j}^{k} P_{k}^{v}
$$

Proof. When $j=1, \ldots, n$, the left and right hand sides of $(2.22)$ are both identically 0. Recall that we have $d\left(P_{j}^{v}\right) \leq s-d(j)<s$ and thus $X_{i} P_{j}^{v}=0$. We prove the claim (2.22) for any $j \leq n$, in particular for $j \leq 0$. As $d(i)=s$, by (ii) in Lemma 2.3 we have $X_{i}=\partial / \partial x_{i}$ on $G=\mathbb{R}^{n}$. Then we have formula 2.18) with $\beta \in \mathcal{I}$ replacing $\beta \in \mathcal{I}_{i}$. We also have formula $(2.19)$, again with $\beta \in \mathcal{I}$ replacing $\beta \in \mathcal{I}_{i}$. Then we are reduced to check identity 2.20 for all $\beta \in \mathcal{I}$.

We claim that, for any $\beta \in \mathcal{I}$, we have

$$
\left[X_{j}, X_{\beta+\mathrm{e}_{i}}\right]=-\left[\left[X_{i}, X_{j}\right], X_{\beta}\right]
$$

We prove 2.23 by induction on the length of $\beta$ and we assume without loss of generality that $i=n$. Notice that the vector field $X_{n}$ commutes with any vector field $X_{k}, k=1, \ldots, n$.

Assume that $\beta_{k} \neq 0$ and $\beta_{h}=0$ for $h>k$. Then, by the Jacobi identity, by $\left[X_{k}, X_{n}\right]=0$, and by formula 2.23 for $\beta-\mathrm{e}_{k}$ replacing $\beta$ (and with $i=n)$, we have

$$
\begin{aligned}
& \left.\left[X_{j}, X_{\beta+\mathrm{e}_{n}}\right]=\left[\left[X_{j}, X_{\beta-\mathrm{e}_{k}}\right], X_{k}\right], X_{n}\right] \\
& =-\left[\left[X_{n},\left[X_{j}, X_{\beta-\mathrm{e}_{k}}\right]\right], X_{k}\right] \\
& =\left[\left[X_{j}, X_{\beta-\mathrm{e}_{k}+\mathrm{e}_{n}}\right], X_{k}\right] \\
& =-\left[\left[\left[X_{n}, X_{j}\right], X_{\beta-\mathrm{e}_{k}}\right], X_{k}\right] \\
& =-\left[\left[X_{n}, X_{j}\right], X_{\beta}\right] \text {, }
\end{aligned}
$$

and we are finished.

Now, using (2.23) the proof can be concluded as in 2.20 - 2.21).

Proof of Theorem 2.2. The proof of (2.15) is a triple nested induction. We fix the vector $v \in \mathbb{R}^{n}$ and we let $P_{j}^{v}=P_{j}$. The claim in Theorem 2.2 reads

$$
X_{i} P_{j}=\sum_{k \leq n} c_{i j}^{k} P_{k} \quad \text { on } G_{\ell} \text { for all } i, \ell=1, \ldots, n \text { and } j \leq n .
$$


"'"LLMV2"final CAG"” — 2018/8/28 — 9:52 — page 734 — \#12

The first induction is descending on $i=1, \ldots, n$ and the base of induction is

$$
X_{n} P_{j}=\sum_{k \leq n} c_{n j}^{k} P_{k} \quad \text { on } G \text { for all } j \leq n
$$

This statement holds by Lemma 2.5 , because $d(n)=s$.

The first inductive assumption is the following

$$
X_{h} P_{j}=\sum_{k \leq n} c_{h j}^{k} P_{k} \quad \text { on } G \text { for all } h>i \text { and for all } j \leq n .
$$

Our goal is to prove identity (2.24) also for $h=i$. The proof of this claim is a descending induction on $j \leq n$. The base of induction for $j=n$ is the following

$$
X_{i} P_{n}=\sum_{k \leq n} c_{i n}^{k} P_{k} \quad \text { on } G
$$

This identity holds because we have $X_{i} P_{n}=0$ ( $P_{n}$ is constant), and $c_{i n}^{k}=0$ for any $i \geq 1$ and for all $k$.

The second inductive assumption is the following

$$
X_{i} P_{h}=\sum_{k \leq n} c_{i h}^{k} P_{k} \quad \text { on } G_{\ell} \text { for all } h>j \text { and for all } \ell=1, \ldots, n
$$

The goal is to prove identity (2.25) also for $h=j$. The proof is a descending induction on $\ell=1, \ldots, i$. The base of induction for $\ell=i$ is the following

$$
X_{i} P_{j}=\sum_{k \leq n} c_{i j}^{k} P_{k} \quad \text { on } G_{i}
$$

that is satisfied by Lemma 2.4 .

The third inductive assumption is

$$
X_{i} P_{j}=\sum_{k \leq n} c_{i j}^{k} P_{k} \quad \text { on } G_{\ell+1}
$$

for $\ell+1 \leq i$. Our goal is to prove identity 2.26) on $G_{\ell}$.

In the following, we can use the three inductive assumptions 2.24)(2.25)-(2.26). 
We prove (2.26) at the point $x=\left(0, \ldots, 0, x_{\ell}, \ldots, x_{n}\right) \in G_{\ell}$. Notice that, by Lemma 2.3 , we have $X_{\ell}=\partial / \partial x_{\ell}$ on $G_{\ell}$. For any $s \in \mathbb{R}$, we let

$$
\begin{aligned}
y_{s} & =\left(0, \ldots, 0, s, x_{\ell+1}, \ldots, x_{n}\right) \in G_{\ell} \\
& =\mathrm{e}^{s X_{\ell}}\left(y_{0}\right) \\
& =\mathrm{e}^{\left(s-x_{\ell}\right) X_{\ell}}(x),
\end{aligned}
$$

where $y_{0}=\left(0, \ldots, 0,0, x_{\ell+1}, \ldots, x_{n}\right) \in G_{\ell+1}$. In particular, we have $y_{x_{\ell}}=x$. Integrating, we obtain the formula

$$
\begin{aligned}
P_{j}(x) & =P_{j}\left(y_{0}\right)+\int_{0}^{x_{\ell}}\left(X_{\ell} P_{j}\right)\left(y_{s}\right) d s \\
& =P_{j}\left(\mathrm{e}^{-x_{\ell} X_{\ell}}(x)\right)+\int_{0}^{x_{\ell}}\left(X_{\ell} P_{j}\right)\left(\mathrm{e}^{\left(s-x_{\ell}\right) X_{\ell}}(x)\right) d s .
\end{aligned}
$$

From the structure (ii) in Lemma 2.3 for the vector field $X_{i}$ with $i>\ell$, we deduce that for any $z=\left(0, \ldots, 0, z_{\ell}, \ldots, z_{n}\right) \in G_{\ell}$ and for any $t \in \mathbb{R}$ we have

$$
\mathrm{e}^{t X_{i}}(z)=\left(0, \ldots, 0, z_{\ell}, *, \ldots, *\right) \in G_{\ell},
$$

and therefore $\mathrm{e}^{t X_{i}}(x)=\left(0, \ldots, 0, x_{\ell}, *, \ldots, *\right)$ and $\mathrm{e}^{t X_{i}}\left(y_{s}\right)=(0, \ldots, 0, s, *$, $\ldots, *)$. Replacing $x$ with $\mathrm{e}^{t X_{i}}(x) \in G_{\ell}$ into the identity (2.27), we get:

$$
\begin{aligned}
P_{j}\left(\mathrm{e}^{t X_{i}}(x)\right)= & P_{j}\left(\mathrm{e}^{-x_{\ell} X_{\ell}} \circ \mathrm{e}^{t X_{i}}(x)\right) \\
& +\int_{0}^{x_{\ell}}\left(X_{\ell} P_{j}\right)\left(\mathrm{e}^{\left(s-x_{\ell}\right) X_{\ell}} \circ \mathrm{e}^{t X_{i}}(x)\right) d s \\
= & P_{j}\left(\mathrm{e}^{-x_{\ell} X_{\ell}} \circ \mathrm{e}^{t X_{i}} \circ \mathrm{e}^{x_{\ell} X_{\ell}}\left(y_{0}\right)\right) \\
& +\int_{0}^{x_{\ell}}\left(X_{\ell} P_{j}\right)\left(\mathrm{e}^{\left(s-x_{\ell}\right) X_{\ell}} \circ \mathrm{e}^{t X_{i}} \circ \mathrm{e}^{-\left(s-x_{\ell}\right) X_{\ell}}\left(y_{s}\right)\right) d s \\
= & P_{j}\left(y_{0} \cdot \exp \left(x_{\ell} X_{\ell}\right) \cdot \exp \left(t X_{i}\right) \cdot \exp \left(-x_{\ell} X_{\ell}\right)\right) \\
& +\int_{0}^{x_{\ell}}\left(X_{\ell} P_{j}\right)\left(y_{s} \cdot \exp \left(-\left(s-x_{\ell}\right) X_{\ell}\right)\right. \\
& \left.\cdot \exp \left(t X_{i}\right) \cdot \exp \left(\left(s-x_{\ell}\right) X_{\ell}\right)\right) d s .
\end{aligned}
$$

For any $z \in G=\mathbb{R}^{n}$, we define the left translation $L_{z}: G \rightarrow G$ and the conjugate mapping $\Gamma_{z}: G \rightarrow G$

$$
L_{z} y=z \cdot y \quad \text { and } \quad \Gamma_{z}(y)=z \cdot y \cdot z^{-1}, \quad y \in G .
$$


With this notation, identity 2.28$)$ reads

$$
\begin{aligned}
P_{j}\left(\mathrm{e}^{t X_{i}}(x)\right)= & P_{j}\left(L_{y_{0}} \Gamma_{\exp \left(x_{\ell} X_{\ell}\right)}\left(\exp \left(t X_{i}\right)\right)\right) \\
& +\int_{0}^{x_{\ell}}\left(X_{\ell} P_{j}\right)\left(L_{y_{s}} \Gamma_{\exp \left(-\left(s-x_{\ell}\right) X_{\ell}\right)}\left(\exp \left(t X_{i}\right)\right)\right) d s
\end{aligned}
$$

and thus

$$
X_{i} P_{j}(x)=\lim _{t \rightarrow 0} \frac{P_{j}\left(\mathrm{e}^{t X_{i}}(x)\right)-P_{j}(x)}{t}=A_{i j}(x)+B_{i j}(x),
$$

where

$$
\begin{aligned}
& A_{i j}(x)=\lim _{t \rightarrow 0} \frac{1}{t}\left[P_{j}\left(L_{y_{0}} \Gamma_{\exp \left(x_{\ell} X_{\ell}\right)}\left(\exp \left(t X_{i}\right)\right)\right)-P_{j}\left(y_{0}\right)\right], \quad \text { and } \\
& B_{i j}(x)=\lim _{t \rightarrow 0} \frac{1}{t} \int_{0}^{x_{\ell}}\left[X_{\ell} P_{j}\left(L_{y_{s}} \Gamma_{\exp \left(-\left(s-x_{\ell}\right) X_{\ell}\right)}\left(\exp \left(t X_{i}\right)\right)\right)-X_{\ell} P_{j}\left(y_{s}\right)\right] d s .
\end{aligned}
$$

In the following we denote by $\operatorname{Ad}_{z}$ the differential of $\Gamma_{z}$. Recall that one has the formula $\operatorname{Ad}_{\exp (Z)}=\mathrm{e}^{\operatorname{ad}(Z)}$, where $\operatorname{ad}(Z)(Y)=[Z, Y]$, see [8, Proposition 1.91]. We have:

$$
\begin{aligned}
A_{i j}(x)= & {\left[\left(d L_{y_{0}}\right)\left(\operatorname{Ad}_{\exp x_{\ell} X_{\ell}}\left(X_{i}\right)\right)\right] P_{j}\left(y_{0}\right) } \\
= & \left\{X_{i}+x_{\ell}\left[X_{\ell}, X_{i}\right]+\frac{1}{2} x_{\ell}^{2}\left[X_{\ell},\left[X_{\ell}, X_{i}\right]\right]+\cdots\right\} P_{j}\left(y_{0}\right) \\
= & \left\{X_{i}-x_{\ell}\left[X_{i}, X_{\ell}\right]+\frac{1}{2} x_{\ell}^{2}\left[\left[X_{i}, X_{\ell}\right], X_{\ell}\right]\right. \\
& \left.-\frac{1}{3 !} x_{\ell}^{3}\left[\left[\left[X_{i}, X_{\ell}\right], X_{\ell}\right], X_{\ell}\right]+\cdots\right\} P_{j}\left(y_{0}\right) \\
= & \left\{X_{i}+\sum_{p=1}^{s} \frac{(-1)^{p}}{p !} x_{\ell}^{p}\left[X_{i}, X_{p \mathrm{e}_{\ell}}\right]\right\} P_{j}\left(y_{0}\right) \\
= & \left\{X_{i}+\sum_{p=1}^{s} \sum_{h=1}^{n} \frac{(-1)^{p}}{p !} x_{\ell}^{p} c_{i, p \mathrm{e}_{\ell}}^{h} X_{h}\right\} P_{j}\left(y_{0}\right) .
\end{aligned}
$$

Notice that for any $p \geq 1$, we have $c_{i, p \mathrm{e}_{\ell}}^{h}=0$ for all $h \leq i$. For $h>i$ we can use the inductive assumption 2.24 and find

$$
X_{h} P_{j}\left(y_{0}\right)=\sum_{k \leq n} c_{h j}^{k} P_{k}\left(y_{0}\right)
$$


Moreover, since $y_{0} \in G_{\ell+1}$, we can also use the inductive assumption 2.26) and find

$$
X_{i} P_{j}\left(y_{0}\right)=\sum_{k \leq n} c_{i j}^{k} P_{k}\left(y_{0}\right)
$$

By 2.29) and 2.30), we obtain the following expression for $A_{i j}$ :

$$
\begin{aligned}
A_{i j}(x)= & \sum_{k \leq n}\left\{c_{i j}^{k}+\sum_{p=1}^{s} \sum_{h=1}^{n} \frac{(-1)^{p}}{p !} x_{\ell}^{p} c_{h j}^{k} c_{i, p \mathrm{e}_{\ell}}^{h}\right\} P_{k}^{v}\left(y_{0}\right) \\
= & \sum_{k \leq n}\left\{c_{i j}^{k}+\sum_{p=1}^{s}\left(\frac{(-1)^{p}}{p !} x_{\ell}^{p} \sum_{h=1}^{n} c_{h j}^{k} c_{i, p \mathrm{e}_{\ell}}^{h}\right)\right\} \\
& \cdot\left[\sum_{\alpha \in \mathcal{I}} \sum_{m \leq n} \frac{(-1)^{|\alpha|}}{\alpha !} c_{k \alpha}^{m} v_{m} y_{0}^{\alpha}\right] .
\end{aligned}
$$

A similar computation for $B_{i j}(x)$ shows that

$$
\begin{aligned}
B_{i j}(x) & =\int_{0}^{x_{\ell}} \lim _{t \rightarrow 0} \frac{1}{t}\left[X_{\ell} P_{j}\left(L_{y_{s}} \Gamma_{\exp \left(-\left(s-x_{\ell}\right) X_{\ell}\right)}\left(\exp \left(t X_{i}\right)\right)\right)-X_{\ell} P_{j}\left(y_{s}\right)\right] d s \\
& =\int_{0}^{x_{\ell}}\left[\left(d L_{y_{s}}\right)\left(\operatorname{Ad}_{\left(x_{\ell}-s\right) X_{\ell}}\left(X_{i}\right)\right)\right]\left(X_{\ell} P_{j}\right)\left(y_{s}\right) d s \\
& =\int_{0}^{x_{\ell}}\left\{X_{i}+\sum_{p=1}^{s} \sum_{u=1}^{n} \frac{(-1)^{p}}{p !}\left(x_{\ell}-s\right)^{p} c_{i, p \mathrm{p}_{\ell}}^{u} X_{u}\right\}\left(X_{\ell} P_{j}\right)\left(y_{s}\right) d s .
\end{aligned}
$$

By (2.16), on $G_{\ell}$ we have the identity

$$
X_{\ell} P_{j}=\sum_{h \leq n} c_{\ell j}^{h} P_{h},
$$

and since $y_{s} \in G_{\ell}$ for all $s$, we obtain

$$
B_{i j}(x)=\int_{0}^{x_{\ell}} \sum_{h \leq n} c_{\ell j}^{h}\left\{X_{i}+\sum_{p=1}^{s} \sum_{u=1}^{n} \frac{(-1)^{p}}{p !}\left(x_{\ell}-s\right)^{p} c_{i, p \mathrm{p}_{\ell}}^{u} X_{u}\right\} P_{h}\left(y_{s}\right) d s .
$$

Notice that $c_{\ell j}^{h}=0$ for $h \leq j$ and $c_{i, p \mathrm{e}_{\ell}}^{u}=0$ for $p \geq 1$ and $u \leq i$. For $h>j$ and $u>i$ we can use the inductive assumptions (2.24) and (2.25). We obtain 
the following expression for $B_{i j}$ :

$$
\begin{aligned}
B_{i j}(x)= & \int_{0}^{x_{\ell}} \sum_{h, k \leq n} c_{\ell j}^{h}\left\{c_{i h}^{k}+\sum_{p=1}^{s} \sum_{u=1}^{n} \frac{(-1)^{p}}{p !}\left(x_{\ell}-s\right)^{p} c_{i, p e_{\ell}}^{u} c_{u h}^{k}\right\} P_{k}\left(y_{s}\right) d s \\
= & \int_{0}^{x_{\ell}}\left[\sum_{h, k \leq n} \sum_{u=1}^{n} \sum_{p=0}^{s} c_{\ell j}^{h} \frac{(-1)^{p}}{p !}\left(x_{\ell}-s\right)^{p} c_{i, p e_{\ell}}^{u} c_{u h}^{k}\right] \\
& \cdot\left[\sum_{\alpha \in \mathcal{I}} \sum_{m \leq n} v_{m} \frac{(-1)^{|\alpha|}}{\alpha !} c_{k, \alpha}^{m} y_{s}^{\alpha}\right] d s .
\end{aligned}
$$

Using the integrals

$$
\begin{aligned}
\int_{0}^{x_{\ell}}\left(x_{\ell}-s\right)^{p} y_{s}^{\alpha} d s & =\int_{0}^{x_{\ell}}\left(x_{\ell}-s\right)^{p} s^{\alpha_{\ell}} x_{1}^{\alpha_{1}} \cdots \widehat{x_{\ell}^{\alpha_{\ell}}} \cdots x_{n}^{\alpha_{n}} d s \\
& =\frac{p ! \alpha_{\ell} !}{\left(\alpha_{\ell}+p+1\right) !} x^{\alpha+(p+1) \mathrm{e}_{\ell}},
\end{aligned}
$$

we finally have

$$
\begin{aligned}
B_{i j}(x) & =\sum_{h, k, m \leq n} \sum_{u=1}^{n} \sum_{p=0}^{s} \sum_{\alpha \in \mathcal{I}_{\ell}} \frac{(-1)^{p+|\alpha|}}{p ! \alpha !} c_{\ell j}^{h} c_{i, p \mathrm{e}_{\ell}}^{u} c_{u h}^{k} c_{k \alpha}^{m} \frac{p ! \alpha_{\ell} !}{\left(\alpha_{\ell}+p+1\right) !} v_{m} x^{\alpha+(p+1) \mathrm{e}_{\ell}} \\
& =-\sum_{h, k, m \leq n} \sum_{u=1}^{n} \sum_{p=0}^{s} \sum_{\alpha \in \mathcal{I}_{\ell}} \frac{(-1)^{\left|\alpha+(p+1) \mathrm{e}_{\ell}\right|}}{\left(\alpha+(p+1) \mathrm{e}_{\ell}\right) !} c_{\ell j}^{h} c_{i, p \mathrm{e}_{\ell}}^{u} c_{u h}^{k} c_{k \alpha}^{m} v_{m} x^{\alpha+(p+1) \mathrm{e}_{\ell}} .
\end{aligned}
$$

Analogously, we have:

$$
A_{i j}(x)=\sum_{h, k, m \leq n} \sum_{p=0}^{s} \sum_{\alpha \in \mathcal{I}_{\ell+1}} \frac{(-1)^{p}}{p !} c_{i, p \mathrm{e}_{\ell}}^{h} c_{h j}^{k} x_{\ell}^{p} \frac{(-1)^{|\alpha|}}{\alpha !} c_{k \alpha}^{m} v_{m} y_{0}^{\alpha},
$$

where the sum ranges over $\alpha \in \mathcal{I}_{\ell+1}$, because $y_{0}=\left(0, \ldots, 0, x_{\ell+1}, \ldots, x_{n}\right)$.

For $\alpha \in \mathcal{I}_{\ell+1}$, we have

$$
\frac{(-1)^{p}}{p !} \frac{(-1)^{|\alpha|}}{\alpha !}=\frac{(-1)^{p+|\alpha|}}{\left(\alpha+p \mathrm{e}_{\ell}\right) !}=c_{\alpha+p \mathrm{e}_{\ell}},
$$

and, moreover, $x_{\ell}^{p} y_{0}^{\alpha}=x^{\alpha+p \mathrm{e}_{\ell}}$. Thus,

$$
A_{i j}(x)=\sum_{k, h, m \leq n} \sum_{p=0}^{s} \sum_{\alpha \in \mathcal{I}_{\ell+1}} c_{\alpha+p \mathrm{e}_{\ell}} c_{i, p \mathrm{e}_{\ell}}^{h} c_{h j}^{k} c_{k \alpha}^{m} v_{m} x^{\alpha+p \mathrm{e}_{\ell}} .
$$


Our goal is to prove that the quantity $X_{i} P_{j}(x)=A_{i j}(x)+B_{i j}(x), x \in$ $G_{\ell}$, equals

$$
C_{i j}(x)=\sum_{k \leq n} c_{i j}^{k} P_{k}(x)=\sum_{k, m \leq n} \sum_{\beta \in I_{\ell}} c_{\beta} c_{i j}^{k} c_{k \beta}^{m} v_{m} x^{\beta} .
$$

The coefficient of $c_{\beta} v_{m} x^{\beta}$ in $C_{i j}(x)$ is

$$
\chi_{C}^{m, \beta}=\sum_{k \leq n} c_{i j}^{k} c_{k \beta}^{m}
$$

The equation $\alpha+p \mathrm{e}_{\ell}=\beta$ with $\alpha_{1}=\cdots=\alpha_{\ell}=0$ implies $p=\beta_{\ell}$ and $\alpha=$ $\beta-p \mathrm{e}_{\ell}=\beta-\beta_{\ell} \mathrm{e}_{\ell}$. Thus, the coefficient of $c_{\beta} v_{m} x^{\beta}$ in $A_{i j}(x)$ is

$$
\chi_{A}^{m, \beta}=\sum_{k, h \leq n} c_{i, \beta_{\ell} \mathrm{e}_{\ell}}^{h} c_{h j}^{k} c_{k, \beta-\beta_{\ell} \mathrm{e}_{\ell}}^{m} .
$$

We compute the coefficient $\chi_{B}^{m, \beta}$ of $c_{\beta} v_{m} x^{\beta}$ in $B_{i j}(x)$. The equation $\alpha+(p+1) \mathrm{e}_{\ell}=\beta$ implies the following: when $\beta_{\ell}=0$ the equation has no solution and thus $\chi_{B}^{m, \beta}=0$; when $\beta_{\ell} \geq 1$ we have $0 \leq p \leq \beta_{\ell}-1$ and $\alpha=$ $\beta-(p+1) \mathrm{e}_{\ell}$, and therefore

$$
\chi_{B}^{m, \beta}=-\sum_{h, k \leq n} \sum_{u=1}^{n} \sum_{p=0}^{\beta_{\ell}-1} c_{\ell j}^{h} c_{i, p \mathrm{e}_{\ell}}^{u} c_{u h}^{k} c_{k, \beta-(p+1) \mathrm{e}_{\ell}}^{m}, \quad \beta_{\ell} \geq 1 .
$$

When $\beta_{\ell}=0$, the identity $\chi_{A}^{m, \beta}+\chi_{B}^{m, \beta}=\chi_{C}^{m, \beta}$ is easily verified.

When $\beta_{\ell} \geq 1$, the identity $\chi_{A}^{m, \beta}+\chi_{B}^{m, \beta}=\chi_{C}^{m, \beta}$ is proved as soon as we verify the following

$$
\sum_{k \leq n} c_{i j}^{k} c_{k \beta}^{m}=\sum_{k, h \leq n} c_{i, \beta_{\ell} \mathrm{e}_{\ell}}^{k} c_{h j}^{k} c_{k, \beta-\beta_{\ell} \mathrm{e}_{\ell}}^{m}-\sum_{h, k \leq n} \sum_{u=1}^{n} \sum_{p=0}^{\beta_{\ell}-1} c_{\ell j}^{h} c_{i, p \mathrm{e}_{\ell}}^{u} c_{u h}^{k} c_{k, \beta-(p+1) \mathrm{e}_{\ell}}^{m} .
$$

Contracting with $X_{m}$ the left hand side of 2.31) we find

$$
\begin{aligned}
\sum_{m, k \leq n} c_{i j}^{k} c_{k \beta}^{m} X_{m} & =\sum_{k \leq n} c_{i j}^{k}\left[X_{k}, X_{\beta}\right]=\left[\left[X_{i}, X_{j}\right], X_{\beta}\right] \\
& =\left[\left[\left[X_{i}, X_{j}\right], X_{\beta_{\ell} \mathrm{e}_{\ell}}\right], X_{\beta-\beta_{\ell} \mathrm{e}_{\ell}}\right] .
\end{aligned}
$$


"'"LLMV2'final CAG"” — 2018/8/28 — 9:52 — page 740 — \#18

Contracting with $X_{m}$ the first term in the right hand side of (2.31) we find

$$
\begin{aligned}
\sum_{k, h, m \leq n} c_{i, \beta_{\ell} \mathrm{e}_{\ell}}^{h} c_{h j}^{k} c_{k, \beta-\beta_{\ell} \mathrm{e}_{\ell}}^{m} X_{m} & =\sum_{k, h \leq n} c_{i, \beta_{\ell} \mathrm{e}_{\ell}}^{h} c_{h j}^{k}\left[X_{k}, X_{\beta-\beta_{\ell} \mathrm{e}_{\ell}}\right] \\
& =\sum_{h=1}^{n} c_{i, \beta_{\ell} \mathrm{e}_{\ell}}^{h}\left[\left[X_{h}, X_{j}\right], X_{\beta-\beta_{\ell} \mathrm{e}_{\ell}}\right] \\
& =\left[\left[\left[X_{i}, X_{\beta_{\ell} \mathrm{e}_{\ell}}\right], X_{j}\right], X_{\beta-\beta_{\ell} \mathrm{e}_{\ell}}\right] .
\end{aligned}
$$

Contracting the second term in the right hand side of 2.31) we find

$$
\begin{aligned}
& -\sum_{h, k, m \leq n} \sum_{u=1}^{n} \sum_{p=0}^{\beta_{\ell}-1} c_{\ell j}^{h} c_{i, p \mathrm{e}_{\ell}}^{u} c_{u h}^{k} c_{k, \beta-(p+1) \mathrm{e}_{\ell}}^{m} X_{m} \\
= & \sum_{p=0}^{\beta_{\ell}-1}\left[\left[\left[\left[X_{i}, X_{p \mathrm{e}_{\ell}}\right],\left[X_{j}, X_{\ell}\right]\right], X_{\left(\beta_{\ell}-p-1\right) \mathrm{e}_{\ell}}\right], X_{\beta-\beta_{\ell} \mathrm{e}_{\ell}}\right] .
\end{aligned}
$$

We used $\beta_{1}=\cdots=\beta_{\ell}=0$.

In 2.32, 2.33), and 2.34, commutators have the same tail $X_{\beta-\beta_{\ell} \mathrm{e}_{\ell}}$. Thus the claim (2.31) follows provided that we prove the following identity:

$$
\begin{aligned}
{\left[\left[X_{i}, X_{j}\right], X_{\beta_{\ell} \mathrm{e}_{\ell}}\right]=} & {\left[\left[X_{i}, X_{\beta_{\ell} \mathrm{e}_{\ell}}\right], X_{j}\right] } \\
& +\sum_{p=0}^{\beta_{\ell}-1}\left[\left[\left[X_{i}, X_{p \mathrm{e}_{\ell}}\right],\left[X_{j}, X_{\ell}\right]\right], X_{\left(\beta_{\ell}-p-1\right)_{\ell}}\right] .
\end{aligned}
$$

We prove 2.35 by induction on $\beta_{\ell} \geq 1$. The base of induction for $\beta_{\ell}=1$ is

$$
\left[\left[X_{i}, X_{j}\right], X_{\ell}\right]=\left[\left[X_{i}, X_{\ell}\right], X_{j}\right]+\left[X_{i},\left[X_{j}, X_{\ell}\right]\right]
$$

and this holds by the Jacobi identity.

By induction, we assume that 2.35 holds for $\beta_{\ell}$ and we prove it for $\beta_{\ell}+1$. We have: 


$$
\begin{aligned}
& {\left[\left[X_{i}, X_{j}\right], X_{\left(\beta_{\ell+1}\right) \mathrm{e}_{\ell}}\right]=\left[\left[\left[X_{i}, X_{j}\right], X_{\beta_{\ell} \mathrm{e}_{\ell}}\right], X_{\ell}\right] } \\
= & {\left[\left[\left[X_{i}, X_{\beta_{\ell} \mathrm{e}_{\ell}}\right], X_{j}\right], X_{\ell}\right]+\sum_{p=0}^{\beta_{\ell}-1}\left[\left[\left[\left[X_{i}, X_{p_{\ell}}\right],\left[X_{j}, X_{\ell}\right]\right], X_{\left(\beta_{\ell}-p-1\right) \mathrm{e}_{\ell}}\right], X_{\ell}\right] } \\
= & {\left[\left[X_{i}, X_{\left(\beta_{\ell}+1\right) \mathrm{e}_{\ell}}\right], X_{j}\right]+\left[\left[X_{i}, X_{\beta_{\ell} \mathrm{e}_{\ell}}\right],\left[X_{j}, X_{\ell}\right]\right] } \\
& +\sum_{p=0}^{\beta_{\ell}-1}\left[\left[\left[X_{i}, X_{p \mathrm{e}_{\ell}}\right],\left[X_{j}, X_{\ell}\right]\right], X_{\left(\beta_{\ell}-p\right) \mathrm{e}_{\ell}}\right] \\
= & {\left[\left[X_{i}, X_{\left(\beta_{\ell}+1\right) \mathrm{e}_{\ell}}\right], X_{j}\right]+\sum_{p=0}^{\beta_{\ell}}\left[\left[\left[X_{i}, X_{p \mathrm{e}_{\ell}}\right],\left[X_{j}, X_{\ell}\right]\right], X_{\left(\beta_{\ell}-p\right) \mathrm{e}_{\ell}}\right] . }
\end{aligned}
$$

This finishes the proof of Theorem 2.2 .

In the next proposition, we list some elementary properties of extremal polynomials that are used in Section 3 .

Proposition 2.6. Extremal polynomials have the following properties.

(i) For all $i=1, \ldots, n$ and $v \in \mathbb{R}^{n}$, we have $P_{i}^{v}(0)=v_{i}$.

(ii) For all $i \in \mathbb{Z}$ with $i \leq 0$ and $v \in \mathbb{R}^{n}$, we have $P_{i}^{v}(0)=0$. $v=0$.

(iii) If $v \in \mathbb{R}^{n}$ is such that $P_{i}^{v}=0$ for all $i=1, \ldots, n$ with $d(i)=1$, then

Proof. (i) For any $i=1, \ldots, n$, we have

$$
P_{i}^{v}(0)=\phi_{i 0}(v)=\sum_{k \leq n} c_{i 0}^{k} v_{k}=v_{i},
$$

because $c_{i 0}^{k}=\delta_{i k}$, the Kronecker symbol.

(ii) This follows as in (i) from the agreement that $v_{k}=0$ for $k \leq 0$.

(iii) Assume that $P_{k}^{v}=0$ when $d(k)=1$. We claim that $P_{k}^{v}=0$ for all $k=1, \ldots, n$. Because of (i), this will imply $v=0$. The proof is by induction on $d(k)$. Assume that $P_{k}^{v}=0$ for all $k=1, \ldots, n$ such that $d(k) \leq \ell$, where $\ell<s$. Take $k=1, \ldots, n$ such that $d(k)=\ell+1$. By the stratification, there are constants $d_{i j}^{k} \in \mathbb{R}, d(i)=1$ and $d(j)=\ell$, such that

$$
X_{k}=\sum_{\begin{array}{c}
d(i)=1 \\
d(j)=\ell
\end{array}} d_{i j}^{k}\left[X_{i}, X_{j}\right]=\sum_{\begin{array}{l}
d(i)=1 \\
d(j)=\ell
\end{array}} \sum_{h \leq n} d_{i j}^{k} c_{i j}^{h} X_{h},
$$


and thus we have the identity

$$
\sum_{\substack{d(i)=1 \\ d(j)=\ell}} d_{i j}^{k} c_{i j}^{h}=\delta_{h k}
$$

From $P_{j}^{v}=0$ for $d(j)=\ell$, it follows that $X_{i} P_{j}^{v}=0$. Now, from Theorem 2.2 and (2.37) we obtain

$$
0=\sum_{\begin{array}{c}
d(i)=1 \\
d(j)=\ell
\end{array}} d_{i j}^{k} X_{i} P_{j}^{v}=\sum_{\begin{array}{l}
d(i)=1 \\
d(j)=\ell
\end{array}} \sum_{h \leq n} d_{i j}^{k} c_{i j}^{h} P_{h}^{v}=P_{k}^{v} .
$$

This finishes the proof.

\section{Characterization of abnormal extremals}

In this section, we prove the theorems about the algebraic characterization of abnormal extremals. The Lie group $G$ is identified with $\mathbb{R}^{n}$ via exponential coordinates as in (2.7), where $X_{1}, \ldots, X_{n}$ is a basis of $\mathfrak{g}=$ $\operatorname{Lie}(G)$ adapted to the stratification. The vector fields $X_{1}, \ldots, X_{r}$ are a basis for $\mathfrak{g}_{1}$ (and thus generators for $\mathfrak{g}$ ). The distribution of $r$-planes $\mathcal{D}(x)=$ $\operatorname{span}\left\{X_{1}(x), \ldots, X_{r}(x)\right\}, x \in G$, is called horizontal distribution of $G$. A Lipschitz curve $\gamma:[0,1] \rightarrow G=\mathbb{R}^{n}$ is $\mathcal{D}$-horizontal, or simply horizontal, if there exists a vector of functions $h=\left(h_{1}, \ldots, h_{r}\right) \in L^{\infty}\left([0,1] ; \mathbb{R}^{r}\right)$ such that

$$
\dot{\gamma}=\sum_{j=1}^{r} h_{j} X_{j}(\gamma), \quad \text { a.e. on }[0,1] \text {. }
$$

The functions $h$ are called controls of $\gamma$ and, when $\gamma:[0,1] \rightarrow \mathbb{R}^{n}$ is given by the coordinates $\gamma=\left(\gamma_{1}, \ldots, \gamma_{n}\right)$, we have $h_{j}=\dot{\gamma}_{j}, j=1, \ldots, r$. This follows from the structure of the vector fields $X_{1}, \ldots, X_{r}$ described in Lemma 2.3 .

Let $g_{x}$ be the quadratic form on $\mathcal{D}(x)$ making $X_{1}, \ldots, X_{r}$ orthonormal. The horizontal length of a horizontal curve $\gamma$ is

$$
L(\gamma)=\left(\int_{0}^{1} g_{\gamma(t)}(\dot{\gamma}(t)) d t\right)^{1 / 2}=\left(\int_{0}^{1}|h(t)|^{2} d t\right)^{1 / 2} .
$$

For any couple of points $x, y \in G$, we can define the function

$$
d(x, y)=\inf \{L(\gamma): \gamma \text { is horizontal, } \gamma(0)=x \text { and } \gamma(1)=y\}
$$


The above set is always nonempty and $d$ is a distance on $G$. A Lipschitz curve $\gamma$ providing the minimum in (3.38) is called a length minimizer.

We denote by $\vartheta_{1}, \ldots, \vartheta_{n}$ the basis of $\mathfrak{g}^{*}$ dual to the basis $X_{1}, \ldots, X_{n}$ of g. A Lipschitz curve of 1 -forms $\lambda \in \operatorname{Lip}\left([0,1] ; \mathfrak{g}^{*}\right)$ is a curve

$$
\lambda=\lambda_{1} \vartheta_{1}+\cdots+\lambda_{n} \vartheta_{n},
$$

where $\lambda_{i}:[0,1] \rightarrow \mathbb{R}, i=1, \ldots, n$, are Lipschitz functions. We call $\lambda_{1}, \ldots, \lambda_{n}$ the coordinates of $\lambda$.

Theorem 3.1. Let $\gamma$ be a length minimizer in $G$. Then there exist a number $\lambda_{0} \in\{0,1\}$ and a Lipschitz curve $\lambda \in \operatorname{Lip}\left([0,1] ; \mathfrak{g}^{*}\right)$ with coordinates $\lambda_{1}, \ldots, \lambda_{n}$ such that:

(i) $\lambda_{0}+|\lambda| \neq 0$ on $[0,1]$;

(ii) $\lambda_{0} \dot{\gamma}_{j}+\lambda_{j}=0$ on $[0,1]$ for all $j=1, \ldots, r$;

(iii) for all $i=1, \ldots, n$, we have

$$
\dot{\lambda}_{i}=-\sum_{k=1}^{n} \sum_{j=1}^{r} c_{i j}^{k} \dot{\gamma}_{j} \lambda_{k} \quad \text { a.e. on }[0,1] \text {, }
$$

where $c_{i j}^{k}$ are the structure constants 2.12.

Theorem 3.1 is a version of Pontryagin Maximum Principle adapted to the present setting. Equations (3.39) are called adjoint equations. We refer to [3, Chapter 12] for a proof of Theorem 3.1 in a more general framework. The version (3.39) of the adjoint equations is derived in [9], Theorem 2.6. The curve $\lambda$ is called a dual curve of $\gamma$.

Definition 3.2. We say that a horizontal curve $\gamma:[0,1] \rightarrow G$ is an extremal if there exist $\lambda_{0} \in\{0,1\}$ and a curve of 1 -forms $\lambda \in \operatorname{Lip}\left([0,1] ; \mathfrak{g}^{*}\right)$ such that i), ii), and iii) in Theorem 3.1 hold.

We say that $\gamma$ is a normal extremal if there exists such a pair $\left(\lambda_{0}, \lambda\right)$ with $\lambda_{0}=1$.

We say that $\gamma$ is an abnormal extremal if there exists such a pair with $\lambda_{0}=0$.

We say that $\gamma$ is a strictly abnormal extremal if $\gamma$ is an abnormal extremal but not a normal one. 
"'LLMV2'final CAG"” — 2018/8/28 — 9:52 — page 744 — \#22

Remark 3.3. If $\gamma$ is an abnormal extremal with dual curve $\lambda$, then we have

$$
\lambda_{1}=\cdots=\lambda_{r}=0 \quad \text { on }[0,1] .
$$

This follows from condition ii) of Theorem 3.1 with $\lambda_{0}=0$.

Theorem 3.4. Let $G$ be a stratified nilpotent Lie group, let $\gamma:[0,1] \rightarrow G$ be a horizontal curve such that $\gamma(0)=0$, and let $\lambda_{1}, \ldots, \lambda_{n}:[0,1] \rightarrow \mathbb{R}$ be Lipschitz functions. The following statements are equivalent:

(A) The functions $\lambda_{1}, \ldots, \lambda_{n}$ solve a.e. the system of equations (3.39).

(B) There exists $v \in \mathbb{R}^{n}$ such that, for all $i=1, \ldots, n$, we have

$$
\lambda_{i}(t)=P_{i}^{v}(\gamma(t)), \quad t \in[0,1],
$$

and in fact $v=\left(\lambda_{1}(0), \ldots, \lambda_{n}(0)\right)$.

Proof. $(\mathrm{B}) \Rightarrow(\mathrm{A})$ Let $\lambda_{1}, \ldots, \lambda_{n}$ be functions on $[0,1]$ defined as in 3.41) for some $v \in \mathbb{R}^{n}$. Then, by Proposition 2.6 we have $v=\left(\lambda_{1}(0), \ldots, \lambda_{n}(0)\right)$. Moreover, by Theorem 2.2 we have, a.e. on $[0,1]$ and for any $i=1, \ldots, n$,

$$
\begin{aligned}
\dot{\lambda}_{i} & =\frac{d}{d t} P_{i}^{v}(\gamma)=\sum_{j=1}^{r} \dot{\gamma}_{j} X_{j} P_{i}^{v}(\gamma) \\
& =\sum_{j=1}^{r} \sum_{k=1}^{n} c_{j i}^{k} \dot{\gamma}_{j} P_{k}^{v}(\gamma)=-\sum_{j=1}^{r} \sum_{k=1}^{n} c_{i j}^{k} \dot{\gamma}_{j} \lambda_{k} .
\end{aligned}
$$

(A) $\Rightarrow$ (B) Let $v=\left(\lambda_{1}(0), \ldots, \lambda_{n}(0)\right) \in \mathbb{R}^{n}$. Then the functions $\lambda_{i}$ and the functions $\mu_{i}=P_{i}^{v}(\gamma), i=1, \ldots, n$, solve the system of differential equation (3.39) with the same initial condition. By the uniqueness of the solution we have $\lambda_{i}=\mu_{i}$.

Next, we define the notion of corank for an abnormal extremal. For any $h \in L^{2}\left([0,1] ; \mathbb{R}^{r}\right)$, let $\gamma^{h}$ be the solution of the problem

$$
\dot{\gamma}^{h}=\sum_{j=1}^{r} h_{j} X_{j}\left(\gamma^{h}\right), \quad \gamma^{h}(0)=x_{0} .
$$

The mapping $\mathcal{E}: L^{2}\left([0,1] ; \mathbb{R}^{r}\right) \rightarrow G=\mathbb{R}^{n}, \mathcal{E}(h)=\gamma^{h}(1)$, is called the endpoint mapping with initial point $x_{0} \in G$. A horizontal curve $\gamma$ starting from 
$x_{0}$ with controls $h$ is an abnormal extremal if and only if there exists $v \in \mathbb{R}^{n}$, $v \neq 0$, such that

$$
\langle d \mathcal{E}(h) w, v\rangle=0
$$

for all $w \in L^{2}\left([0,1] ; \mathbb{R}^{r}\right)$. Here, $d \mathcal{E}(h)$ is the differential of $\mathcal{E}$ at the point $h$.

Definition 3.5. The corank of an abnormal extremal $\gamma:[0,1] \rightarrow \mathbb{R}^{n}$ with controls $h$ is the integer $\operatorname{corank}(\gamma)=n-\operatorname{dim}(\operatorname{Im} d \mathcal{E}(h)) \geq 1$.

The corank of an abnormal extremal $\gamma:[0,1] \rightarrow G$ is the integer $m \geq 1$ such that there exist precisely $m$ linearly independent dual curves $\lambda^{1}, \ldots, \lambda^{m} \in$ $\operatorname{Lip}\left([0,1] ; \mathfrak{g}^{*}\right)$ each solving the system of adjoint equations $(3.39)$ with initial conditions $\lambda^{1}(0), \ldots, \lambda^{m}(0) \in \mathbb{R}^{n}$ that are orthogonal to the image of the differential of the end-point mapping.

Definition 3.6. Let $G=\mathbb{R}^{n}$ be a stratified nilpotent Lie group of rank $r$. For any $v \in \mathbb{R}^{n}, v \neq 0$, we call the set

$$
Z_{v}=\left\{x \in \mathbb{R}^{n}: P_{j}^{v}(x)=0 \text { for all } j \in \mathbb{Z} \text { with } j \leq r\right\}
$$

an abnormal variety of $G$ of corank 1 .

For linearly independent vectors $v_{1}, \ldots, v_{m} \in \mathbb{R}^{n}, m \geq 2$, we call the set $Z_{v_{1}} \cap \cdots \cap Z_{v_{m}}$ an abnormal variety of $G$ of corank $m$.

Remark 3.7. The abnormal variety $Z_{v}$ is the intersection of the zero sets of all the polynomials $P_{j}^{v}$ with $j \leq r$. In this intersection, also indexes $j \leq 0$ are involved. With this respect, Definition 3.6 differs from the analogous definition in [9].

Theorem 3.8. Let $G=\mathbb{R}^{n}$ be a stratified nilpotent Lie group and let $\gamma$ : $[0,1] \rightarrow G$ be a horizontal curve with $\gamma(0)=0$. The following statements are equivalent:

(A) The curve $\gamma$ is an abnormal extremal with $\operatorname{corank}(\gamma) \geq m \geq 1$.

(B) There exist $m \geq 1$ linearly independent vectors $v_{1}, \ldots, v_{m} \in \mathbb{R}^{n}$ such that $\gamma(t) \in Z_{v_{1}} \cap \cdots \cap Z_{v_{m}}$ for all $t \in[0,1]$.

Proof. (A) $\Rightarrow$ (B) Let $\gamma$ be an abnormal extremal and let $\lambda=\lambda_{1} \vartheta_{1}+\cdots+$ $\lambda_{n} \vartheta_{n}$ be a Lipschitz curve of 1 -forms with coordinates $\lambda_{1}, \ldots, \lambda_{n}$ solving the system of differential equations (3.39) and such that $\lambda \neq 0$ point-wise. 
By Theorem 3.4, there is a $v \in \mathbb{R}^{n}, v \neq 0$, such that $\lambda_{i}=P_{i}^{v}(\gamma)$ for all $i=1, \ldots, n$. In particular, there holds $\left(\lambda_{1}(0), \ldots, \lambda_{n}(0)\right)=v$. For abnormal extremals, we have $\lambda_{1}=\cdots=\lambda_{r}=0$ on $[0,1]$, i.e., $\gamma(t) \in\left\{x \in \mathbb{R}^{n}: P_{j}^{v}(x)=\right.$ 0 for all $j=1, \ldots, r\}$ for all $t \in[0,1]$.

We claim that $P_{j}^{v}(\gamma)=0$ also for all $j \in \mathbb{Z}$ with $j \leq 0$. The proof is by induction on $d(j)$. The base of induction for $d(j)=1$ is proved above. By induction, assume that $P_{k}^{v}(\gamma)=0$ for all $k \in \mathbb{Z}$ such that $d(i)<d(k) \leq 1$. As in (3.42), by Theorem 2.2 we have

$$
\frac{d}{d t} P_{i}^{v}(\gamma)=-\sum_{j=1}^{r} \sum_{k \leq n} c_{i j}^{k} \dot{\gamma}_{j} P_{k}^{v}(\gamma)=0
$$

because $c_{i j}^{k}=0$ for $d(k) \leq d(i)$, for any $j=1, \ldots, r$. It follows that $P_{i}^{v}(\gamma)=$ $P_{i}^{v}(\gamma(0))$ is constant. By Proposition 2.6 and $\gamma(0)=0$, it is the zero constant. This shows that $\gamma(t) \in Z_{v}$ for all $t \in[0,1]$.

From the above argument, we conclude that if $\operatorname{corank}(\gamma) \geq m$ then there exist at least $m$ linearly independent vectors $v_{1}, \ldots, v_{m} \in \mathbb{R}^{n}$ such that $\gamma(t) \in Z_{v_{i}}$ for all $t \in[0,1]$ and $i=1, \ldots, m$.

(B) $\Rightarrow$ (A) Let $v_{1}, \ldots, v_{m} \in \mathbb{R}^{n}$ be linearly independent vectors such that (B) holds. For any $v=v_{1}, \ldots, v_{m}$, the curve $\lambda=\lambda_{1} \vartheta_{1}+\cdots+\lambda_{n} \vartheta_{n}$ with coordinates $\lambda_{i}=P_{i}^{v}(\gamma)$ satisfies the system (3.39), by Theorem 3.4. Moreover, we have $\lambda_{1}=\cdots=\lambda_{r}=0$ because $\gamma$ is an abnormal extremal. From $v \neq 0$, it follows that $\left(\lambda_{1}(0), \ldots, \lambda_{n}(0)\right)=v \neq 0$, and from the uniqueness of the solution to 3.39 with initial condition, it follows that $\lambda \neq 0$ point-wise on $[0,1]$. As $v_{1}, \ldots, v_{m}$ are linearly independent, then the corresponding curves $\lambda$ are also linearly independent. We conclude $\operatorname{corank}(\gamma) \geq m$.

Remark 3.9. In the rank 2 case, an abnormal extremal $\gamma$ satisfies $\lambda_{1}=$ $P_{1}^{v}(\gamma)=0$ and $\lambda_{2}=P_{2}^{v}(\gamma)=0$. When $\gamma$ is not a constant curve, these equations imply that also $\lambda_{3}=0$. Indeed, the equations 3.39 for $\lambda_{1}$ and $\lambda_{2}$ are

$$
\dot{\lambda}_{1}=\dot{\gamma}_{2} \lambda_{3} \quad \text { and } \quad \dot{\lambda}_{2}=-\dot{\gamma}_{1} \lambda_{3}
$$

Thus, if $\dot{\gamma}_{1}^{2}+\dot{\gamma}_{2}^{2} \neq 0$, it must be $\lambda_{3}=0$.

\section{The abnormal set in the free group of rank 2 and step 4}

In this section, we present a method to compute a set containing all abnormal extremals passing through one point. The method works when each 
abnormal extremal is in the zero sets of a sufficiently large number of extremal polynomials. This is the case when the prolongation is sufficiently large. In order to make the presentation clear, we focus on the free group of rank 2 and step 4 . The key point is to show that a certain polynomial is nontrivial, see Theorem 4.1 below.

Let $\mathfrak{g}=\mathfrak{g}_{1} \oplus \cdots \oplus \mathfrak{g}_{4}$ be the free nilpotent Lie algebra with rank $r=2$ and step $s=4$. This algebra is 8 -dimensional. Let $X_{1}, \ldots, X_{8}$ be a Hall basis of $\mathfrak{g}$ (see [7] and [9]). This basis satisfies the following structure relations

$$
\begin{array}{ll}
X_{3}=\left[X_{2}, X_{1}\right], & X_{4}=\left[X_{3}, X_{1}\right], \quad X_{5}=\left[X_{3}, X_{2}\right], \\
X_{6}=\left[X_{4}, X_{1}\right], \quad X_{7}=\left[X_{4}, X_{2}\right], \quad X_{8}=\left[X_{5}, X_{2}\right] .
\end{array}
$$

In the Grayson-Grossman model [5], $X_{1}, \ldots, X_{8}$ can be identified with vector fields in $\mathbb{R}^{8}$ where

$$
\begin{aligned}
X_{1}= & \frac{\partial}{\partial x_{1}}, \\
X_{2}= & \frac{\partial}{\partial x_{2}}-x_{1} \frac{\partial}{\partial x_{3}}+\frac{x_{1}^{2}}{2} \frac{\partial}{\partial x_{4}}+x_{1} x_{2} \frac{\partial}{\partial x_{5}} \\
& -\frac{x_{1}^{3}}{6} \frac{\partial}{\partial x_{6}}-\frac{x_{1}^{2} x_{2}}{2} \frac{\partial}{\partial x_{7}}-\frac{x_{1} x_{2}^{2}}{2} \frac{\partial}{\partial x_{8}} .
\end{aligned}
$$

The Tanaka prolongation $\operatorname{Prol}(\mathfrak{g})$ is finite dimensional and by Theorem 1 in [21, we have

$$
\operatorname{Prol}(\mathfrak{g})=\mathfrak{g}_{0} \oplus \mathfrak{g}
$$

where $\mathfrak{g}_{0}$ is the vector space of strata preserving derivations of $\mathfrak{g}$. In fact, $\mathfrak{g}_{0}$ is isomorphic to $\operatorname{End}\left(\mathfrak{g}_{1}\right)$ and thus $\mathfrak{g}_{0}$ is 4 -dimensional, because $\operatorname{dim}\left(\mathfrak{g}_{1}\right)=2$. We extend the basis for $\mathfrak{g}$ to a basis $X_{-3}, X_{-2}, \ldots, X_{8}$ of $\operatorname{Prol}(\mathfrak{g})$ and we denote by $c_{j \alpha}^{k}$, with $j=-3,-2, \ldots, 8, k=1, \ldots, 8$, and $\alpha \in \mathcal{I}=\mathbb{N}^{8}$, the generalized structure constants of $\operatorname{Prol}(\mathfrak{g})$.

Let $G$ be the free nilpotent Lie group of rank $r=2$ and step $s=4$. Via exponential coordinates of the second type, $G$ is diffeomorphic to $\mathbb{R}^{8}$. The extremal polynomials in $\mathbb{R}^{8}$ associated with the Tanaka prolongation of $\mathfrak{g}=\operatorname{Lie}(G)$ are, for any fixed $v \in \mathbb{R}^{8}$,

$$
P_{j}^{v}(x)=\sum_{\alpha \in \mathcal{I}} \frac{(-1)^{|\alpha|}}{\alpha !} \sum_{k=1}^{8} c_{j \alpha}^{k} v_{k} x^{\alpha}=\sum_{k=1}^{8} v_{k} Q_{j k}(x), \quad x \in \mathbb{R}^{8},
$$


where $Q_{j k}$ are the polynomials in $\mathbb{R}^{8}$

$$
Q_{j k}(x)=\sum_{\alpha \in \mathcal{I}} \frac{(-1)^{|\alpha|}}{\alpha !} c_{j \alpha}^{k} x^{\alpha}, \quad x \in \mathbb{R}^{8} .
$$

Above, we have $j=-3,-2, \ldots, 8$ and $k=1, \ldots, 8$. Notice that $c_{j \alpha}^{k}=0$ if $d(j)+d(\alpha) \neq d(k)$. It follows that $Q_{j k}$ is a polynomial with homogeneous degree $d(k)-d(j)$.

Let $v \in \mathbb{R}^{8}$ be a vector such that $v_{1}=v_{2}=v_{3}=0$. The first three polynomials $P_{1}^{v}, P_{2}^{v}$, and $P_{3}^{v}$ are the following

$$
\begin{aligned}
P_{1}^{v}(x)= & v_{4} x_{3}-v_{5} \frac{x_{2}^{2}}{2}+v_{6} x_{4}+v_{7} x_{5}+v_{8} \frac{x_{2}^{3}}{6} \\
P_{2}^{v}(x)= & v_{4} \frac{x_{1}^{2}}{2}+\left(x_{3}+x_{1} x_{2}\right) v_{5}-\frac{x_{1}^{3}}{6} v_{6}+\left(x_{4}-\frac{x_{1}^{2} x_{2}}{2}\right) v_{7} \\
& +\left(x_{5}-\frac{x_{1} x_{2}^{2}}{2}\right) v_{8}, \\
P_{3}^{v}(x)= & -v_{4} x_{1}-v_{5} x_{2}+v_{6} \frac{x_{1}^{2}}{2}+v_{7} x_{1} x_{2}+v_{8} \frac{x_{2}^{2}}{2} .
\end{aligned}
$$

These polynomials can be computed using the structure relations (4.44) and formulas (4.46).

In order to compute the four polynomials $P_{-3}^{v}, P_{-2}^{v}, P_{-1}^{v}$, and $P_{0}^{v}$ associated with the stratum $\mathfrak{g}_{0}$ of $\operatorname{Prol}(\mathfrak{g})$, we have to choose a basis $X_{-3}, X_{-2}$, $X_{-1}, X_{0}$ of $\mathfrak{g}_{0}$. We can identify $\mathfrak{g}_{0}$ with $\operatorname{End}\left(\mathfrak{g}_{1}\right)$, and $\mathfrak{g}_{1}$ with $\mathbb{R}^{2}$ via the basis $X_{1}, X_{2}$. Hence, we can make the following choice:

$$
\begin{array}{ll}
X_{-3}=\left(\begin{array}{ll}
0 & 1 \\
0 & 0
\end{array}\right), & X_{-2}=\left(\begin{array}{ll}
0 & 0 \\
0 & 1
\end{array}\right), \\
X_{-1}=\left(\begin{array}{ll}
1 & 0 \\
0 & 0
\end{array}\right), & X_{0}=\left(\begin{array}{ll}
0 & 0 \\
1 & 0
\end{array}\right) .
\end{array}
$$

The commutator $\left[X_{j}, X_{i}\right]$, with $j=-3, \ldots, 0$ and $i=1,2$, is identified with $X_{j}\left(X_{i}\right)$ where $X_{j}$ is a linear operator on $\mathfrak{g}_{1}$. The polynomials associated with this basis are the following 


$$
\begin{aligned}
P_{-3}^{v}(x)= & v_{4}\left(x_{3} x_{2}+x_{5}\right)-v_{5} \frac{x_{2}^{3}}{6}+v_{6}\left(x_{4} x_{2}+x_{7}\right) \\
& +v_{7}\left(x_{5} x_{2}+2 x_{8}\right)+v_{8} \frac{x_{2}^{4}}{24}, \\
P_{-2}^{v}(x)= & v_{4} x_{4}+v_{5}\left(x_{2} x_{3}+2 x_{5}\right)+v_{6} x_{6}+v_{7}\left(x_{2} x_{4}+2 x_{7}\right) \\
& +v_{8}\left(x_{2} x_{5}+3 x_{8}\right), \\
P_{-1}^{v}(x)= & v_{4}\left(x_{1} x_{3}+2 x_{4}\right)+v_{5}\left(x_{5}-\frac{x_{1} x_{2}^{2}}{2}\right)+v_{6}\left(x_{1} x_{4}+3 x_{6}\right) \\
& +v_{7}\left(x_{1} x_{5}+2 x_{7}\right)+v_{8}\left(\frac{x_{1} x_{2}^{3}}{6}+x_{8}\right), \\
P_{0}^{v}(x)= & v_{4} \frac{x_{1}^{3}}{6}+v_{5}\left(\frac{x_{1}^{2} x_{2}}{2}+x_{1} x_{3}+x_{4}\right)-v_{6} \frac{x_{1}^{4}}{24} \\
& +v_{7}\left(-\frac{x_{1}^{3} x_{2}}{6}+x_{1} x_{4}+2 x_{6}\right) \\
& +v_{8}\left(-\frac{x_{1}^{2} x_{2}^{2}}{4}+x_{1} x_{5}+x_{7}\right) .
\end{aligned}
$$

These formulas can be checked in the following way. According to 2.15), the polynomials 4.49 have to satisfy the structure identities

$$
\begin{aligned}
& X_{1} P_{-3}^{v}=0, \quad X_{1} P_{-2}^{v}=0, \quad X_{1} P_{-1}^{v}=P_{1}^{v}, \quad X_{1} P_{0}^{v}=P_{2}^{v}, \\
& X_{2} P_{-3}^{v}=P_{1}^{v}, \quad X_{2} P_{-2}^{v}=P_{2}^{v}, \quad X_{2} P_{-1}^{v}=0, \quad X_{2} P_{0}^{v}=0 .
\end{aligned}
$$

Notice that we have the structure constants $c_{-3,1}^{k}=c_{-2,1}^{k}=c_{-1,2}^{k}=c_{0,2}^{k}=0$, $c_{-3,2}^{k}=c_{-1,1}^{k}=\delta_{1 k}, c_{-2,2}^{k}=c_{0,1}^{k}=\delta_{2 k}, k=1,2$. Along with the condition $P_{j}^{v}(0)=0, j=-3, \ldots, 0$, the identities 4.50 uniquely determine the polynomials. Now, by a direct computation based on 4.45, it can be checked that the polynomials 4.49 do satisfy 4.50 .

The polynomials (4.49) can also be computed using the following algorithm. Let $\gamma:[0,1] \rightarrow \mathbb{R}^{8}$ be a horizontal curve such that $\gamma(0)=0$ and, for $i, j=1,2$, let

$$
B_{i j}^{v}(t)=\int_{0}^{t} P_{i}^{v}(\gamma) \dot{\gamma}_{j} d s, \quad t \in[0,1]
$$

Using the formulas 4.48 for $P_{1}^{v}$ and $P_{2}^{v}$, the integrals in the right hand side can be computed. Using the explicit formulas for the coefficients of the vector field $X_{2}$ in 4.45 , the resulting functions can be shown to be polynomials 
of the coordinates of $\gamma(t)$. Namely, along $\gamma$ we have $B_{12}^{v}=P_{-3}^{v}, B_{22}^{v}=P_{-2}^{v}$, $B_{11}^{v}=P_{-1}^{v}$, and $B_{21}^{v}=P_{0}^{v}$.

Let $\gamma:[0,1] \rightarrow G=\mathbb{R}^{8}$ be an abnormal extremal such that $\gamma(0)=0$. By Theorem 3.8 (see also Remark 3.9) there exists $v \in \mathbb{R}^{8}$, with $v \neq 0$, such that, for all $j=-3,-2, \ldots, 3$, we have

$$
P_{j}^{v}(\gamma(t))=0, \quad t \in[0,1] .
$$

In particular, $v=\left(v_{1}, \ldots, v_{8}\right)$ satisfies $v_{1}=v_{2}=v_{3}=0$ (because $\gamma(0)=0$ ).

For each $j=-3, \ldots, 3$, we define the following 5 -dimensional vector of polynomials

$$
Q_{j}(x)=\left(Q_{j 4}(x), \ldots, Q_{j 8}(x)\right),
$$

where $Q_{j k}$ are defined in (4.47). By (4.46), identity (4.51) reads

$$
\sum_{k=4}^{8} v_{k} Q_{j k}(\gamma(t))=0, \quad t \in[0,1] .
$$

In other words, along the curve $\gamma$ the seven 5-dimensional vectors $Q_{-3}(x)$, $\ldots, Q_{3}(x)$ are orthogonal to the nonzero vector $\left(v_{4}, \ldots, v_{8}\right) \in \mathbb{R}^{5}$. It follows that the $7 \times 5$ matrix

$$
Q(x)=\left(\begin{array}{ccc}
Q_{-3,4}(x) & \cdots & Q_{-3,8}(x) \\
\vdots & & \vdots \\
Q_{3,4}(x) & \cdots & Q_{3,8}(x)
\end{array}\right)
$$

has rank at most 4 along the curve $\gamma$.

Let $M_{1}(x), \ldots, M_{21}(x)$ be the 21 different $5 \times 5$ minors of $Q(x)$ and define the determinant functions

$$
f_{k}(x)=\operatorname{det}\left(M_{k}(x)\right), \quad k=1, \ldots, 21, \quad x \in \mathbb{R}^{8} .
$$

It can be proved that the functions $f_{1}, \ldots, f_{21}$ are homogeneous polynomials. By the discussion above, we have

$$
f_{k}(\gamma(t))=0 \quad \text { for all } t \in[0,1] \text { and for all } k=1, \ldots, 21 .
$$

The polynomials $f_{1}, \ldots, f_{21}$ do not depend on $v$.

Theorem 4.1. Let $G=\mathbb{R}^{8}$ be the free nilpotent Lie group of rank 2 and step 4 . There exists a nonzero homogeneous polynomial $Q: \mathbb{R}^{8} \rightarrow \mathbb{R}$ such that 
any abnormal curve $\gamma:[0,1] \rightarrow \mathbb{R}^{8}=G$ with $\gamma(0)=0$ satisfies $Q(\gamma(t))=0$ for all $t \in[0,1]$.

Proof. It is enough to show that at least one of the polynomials $f_{1}, \ldots, f_{21}$ in 4.52 is nonzero. Let us consider the polynomial with least degree

$$
Q(x)=\operatorname{det} M(x)=\operatorname{det}\left(\begin{array}{ccc}
Q_{-1,4}(x) & \cdots & Q_{-1,8}(x) \\
Q_{0,4}(x) & \cdots & Q_{0,8}(x) \\
\vdots & & \vdots \\
Q_{3,4}(x) & \cdots & Q_{3,8}(x)
\end{array}\right)
$$

The homogeneous degree of $Q$ is 14 . We highlight the anti-diagonal of the matrix $M$ :

$$
M(x)=\left(\begin{array}{ccccc}
* & * & * & * & x_{1} x_{2}^{3} / 6+x_{8} \\
* & * & * & -x_{1}^{3} x_{2} / 6+x_{1} x_{4}+2 x_{6} & * \\
* & * & x_{4} & * & * \\
* & x_{1} x_{2}+x_{3} & * & * & * \\
-x_{1} & * & * & * & *
\end{array}\right) .
$$

Upon inspection of the polynomials $P_{-1}^{v}, \ldots, P_{3}^{v}$ in (4.48) and (4.49), we observe the following facts. The variable $x_{8}$ appears in the entry $M_{i j}$ only when $i=j=5$. When $i, j \leq 4$, the variable $x_{6}$ appears only in the entry $M_{i j}$ with $i=j=4$. When $i, j \leq 3$, the variable $x_{4}$ appears only in the entry $M_{i j}$ with $i=j=3$. When $i, j \leq 2$, the variable $x_{3}$ appears only in the entry $M_{i j}$ with $i, j=2$. Finally, we have $M_{11}=-x_{1}$. It follows that

$$
Q(x)=-2 x_{1} x_{3} x_{4} x_{6} x_{8}+R(x),
$$

where $R(x)$ is a polynomial that does not contain the monomial $x_{1} x_{3} x_{4} x_{6} x_{8}$. This proves that $Q \neq 0$.

Remark 4.2. Any abnormal curve passing through 0 is in the intersection of the zero sets of the 21 polynomials 4.52 in $\mathbb{R}^{8}$. Even though all these polynomials are explicitly computable, the precise structure of this intersection is not clear.

For any $v \in \mathbb{R}^{8}$ with $v \neq 0$ and $v_{1}=v_{2}=v_{3}$, the equation $P_{3}^{v}(x)=0$ determines two (in some exceptional cases four) different abnormal extremals $\gamma^{v}$ parametrized by arc-length and such that $\gamma^{v}(0)=0$. The mapping $v \mapsto$ $\gamma^{v}(1)$ seems to parametrize a subset of $\mathbb{R}^{8}$ that is at most 5-dimensional. The abnormal set of $G=\mathbb{R}^{8}$ is then presumably 6-dimensional, while in 
Theorem 4.1 abnormal extremals are shown to be in a set with dimension less than or equal to 7 .

\section{Spiral-like Goh extremals}

The shortening technique introduced in [12] has two steps: a curve with a corner at a given point is blown up; the limit curve obtained in this way is shown not to be length minimizing. This provides an "almost- $C$ " regularity result for sub-Riemannian length minimizing curves. The technique, however, fails when, in the blow up at the singular point, the curve has no proper angle. Here, we show that there do exist Goh extremals of this kind. The example is a generalization of [9, Example 6.4].

Let $G$ be an $n$-dimensional stratified nilpotent Lie group with Lie algebra $\mathfrak{g}=\mathfrak{g}_{1} \oplus \cdots \oplus \mathfrak{g}_{s}$, where $s \geq 3$ is step of the group. An abnormal extremal $\gamma:[0,1] \rightarrow G$ with dual curve $\lambda=\lambda_{1} \vartheta_{1}+\cdots+\lambda_{n} \vartheta_{n}$ is said to be a Goh extremal if $\lambda_{i}=0$ for all $i=1, \ldots, n$ such that $d(i)=1$ or $d(i)=2$. By Theorem 3.4, a horizontal curve $\gamma:[0,1] \rightarrow G$ with $\gamma(0)=0$ is a Goh extremal precisely when there exists $v \in \mathbb{R}^{n}, v \neq 0$, such that

$$
P_{i}^{v}(\gamma(t))=0, \quad \text { for all } t \in[0,1] \text { and } d(i) \in\{1,2\}
$$

By Theorem 2.2, the condition $P_{i}^{v}(\gamma)=0$ for $d(i)=2$ is equivalent to 5.53 .

Let $F$ be the free nilpotent Lie group of rank 3 and step 4 and consider the direct product $G=F \times F$. As $F$ is diffeomorphic to $\mathbb{R}^{32}$, then $G$ is a stratified Lie group of rank 6 and step 4 diffeomorphic to $\mathbb{R}^{64}$. We fix a basis $X_{1}, \ldots, X_{64}$ of $\operatorname{Lie}(G)$ adapted to the stratification and we identify $G$ with $\mathbb{R}^{64}$ via exponential coordinates of the second type. We reorder and relabel the basis as $Y_{1}, \ldots, Y_{32}, Z_{1}, \ldots, Z_{32}$, where $Y_{1}, \ldots, Y_{32}$ is an adapted basis of a first copy of $\operatorname{Lie}(F)$ and $Z_{1}, \ldots, Z_{32}$ is an adapted basis of a second copy of $\operatorname{Lie}(F)$. We denote the corresponding coordinates on $G$ by $(y, z) \in \mathbb{R}^{64}$ with $y, z \in \mathbb{R}^{32}$.

Notice that we have $\left[Y_{i}, Z_{j}\right]=0$ for all $i, j=1, \ldots, 32$. Then any abnormal polynomial $P_{i}^{v}(x)$ of $G, i=1, \ldots, 64$ and $v \in \mathbb{R}^{64}$, splits as

$$
P_{i}^{v}(x)=Q_{j}^{v_{y}}(y)+Q_{k}^{v_{z}}(z), \quad x=(y, z),
$$

for some $j, k=1, \ldots, 32$ and $v_{y}, v_{z} \in \mathbb{R}^{32}$, where $Q_{1}^{v}, \ldots, Q_{32}^{v}, v \in \mathbb{R}^{32}$, are the extremal polynomials of $F$. In particular, for $i=4,5,6$ we have the 
polynomials:

$$
\begin{aligned}
Q_{4}^{v}(y)= & v_{4}-y_{1} v_{7}-y_{2} v_{8}-y_{3} v_{9}+\frac{y_{1}^{2}}{2} v_{15}+y_{1} y_{2} v_{16}+y_{1} y_{3} v_{17}+\frac{y_{2}^{2}}{2} v_{18} \\
& +y_{2} y_{3} v_{19}+\frac{y_{3}^{2}}{2} v_{20}+y_{5} v_{30}+y_{6} v_{31} \\
Q_{5}^{v}(y)= & v_{5}-y_{1} v_{10}-y_{2} v_{11}-y_{3} v_{12}+\frac{y_{1}^{2}}{2} v_{21}+y_{1} y_{2} v_{22}+y_{1} y_{3} v_{23}+\frac{y_{2}^{2}}{2} v_{24} \\
& +y_{2} y_{3} v_{25}+\frac{y_{3}^{2}}{2} v_{26}-y_{4} v_{30}+y_{6} v_{32} \\
Q_{6}^{v}(y)= & v_{6}+y_{1}\left(v_{9}-v_{11}\right)-y_{2} v_{13}-y_{3} v_{14}-\frac{y_{1}^{2}}{2} v_{17}-y_{1} y_{2} v_{19}-y_{1} y_{3} v_{20} \\
& +\frac{y_{1}^{2}}{2} v_{22}+y_{1} y_{2} v_{24}+y_{1} y_{3} v_{25}+\frac{y_{3}^{2}}{2} v_{29}+y_{1}^{2} v_{30} \\
& +\left(y_{1} y_{2}-y_{4}\right) v_{31}-y_{5} v_{32} .
\end{aligned}
$$

We omit the deduction of these formulas.

Let $v, w \in \mathbb{R}^{32}$ be such that $v_{i}=w_{i}=0$ for all $i=1, \ldots, 6$ and $|v|+$ $|w| \neq 0$. By Theorem 3.4, any horizontal curve $\gamma=\left(\gamma_{y}, \gamma_{z}\right):[0,1] \rightarrow G$ such that $\gamma(0)=0$ and with support contained in the algebraic set

$$
\Sigma_{v, w}=\left\{(y, z) \in \mathbb{R}^{64}: Q_{i}^{v}(y)=Q_{i}^{w}(z)=0 \text { for } i=4,5,6\right\}
$$

is a Goh extremal. Notice that, by (2.15), $Q_{i}^{v}\left(\gamma_{y}\right)=Q_{i}^{w}\left(\gamma_{z}\right)=0$ for $i=4,5,6$ implies $Q_{i}^{v}\left(\gamma_{y}\right)=Q_{i}^{w}\left(\gamma_{z}\right)=0$ also for $i=1,2,3$. We choose $v \in \mathbb{R}^{32}$ such that $v_{7}=1, v_{18}=2$, and $v_{j}=0$ otherwise. Then we have

$$
Q_{4}^{v}(y)=y_{2}^{2}-y_{1}, \quad Q_{5}^{v}(y)=Q_{6}^{v}(y)=0, \quad y=\left(y_{1}, \ldots, y_{32}\right) .
$$

For any pair of Lipschitz functions $\phi, \psi:[-1,1] \rightarrow \mathbb{R}$ with $\phi(0)=\psi(0)=$ 0 , the horizontal curve $\gamma=\left(\gamma_{y}, \gamma_{z}\right):[-1,1] \rightarrow \mathbb{R}^{64}$ defined by $\gamma_{y}(t)=\left(t^{2}, t\right.$, $\phi(t), *, \ldots, *)$ and $\gamma_{z}(t)=\left(t^{2}, t, \psi(t), *, \ldots, *\right)$ has support contained in $\Sigma_{v, v}$ and therefore it is a Goh extremal.

In particular, as in [4], we can choose

$$
\phi(t)=t \cos (\log (1-\log |t|)), \quad \psi(t)=t \sin (\log (1-\log |t|)), \quad t \in[-1,1],
$$

where $\phi(0)=\psi(0)=0$ in the limit sense. The curve $\gamma$ is a kind of rectifiable spiral. Notice that $\max \left(\left|\phi^{\prime}(t)\right|,\left|\psi^{\prime}(t)\right|\right) \leq 2$ for all $t \in(0,1)$. Moreover, for any $\alpha \in[0,2 \pi)$ there exists a positive and infinitesimal sequence $\left(\lambda_{k}\right)_{k \in \mathbb{N}}$ 
such that

$$
\lim _{k \rightarrow \infty} \frac{1}{\lambda_{k}}\left(\phi\left(\lambda_{k} t\right), \psi\left(\lambda_{k} t\right)\right)=t(\cos \alpha, \sin \alpha), \quad t \in \mathbb{R} .
$$

Vice versa, if the limit in the left hand side does exist for some infinitesimal sequence $\left(\lambda_{k}\right)_{k \in \mathbb{N}}$, then it is of the given form for some $\alpha \in[0,2 \pi)$.

The curve $\gamma$ is not $C^{1}$ at $t=0$ and all its tangents at $t=0$ are lines. It is a natural question whether the Goh extremal $\gamma$ is length minimizing or not. So far we were not able to answer this question. The unique result on the nonminimality of extremals with singular points is the removability of corners, see [12]. This was obtained through a shortening technique that consists of two steps. In a first step, the curve and the structure are blown up at the singular point getting in the limit a "canonical flat corner" (the union of two perpendicular half lines). In a second step, the flat corner is proved not to be length minimizing by constructing a shorter curve cutting the corner and adjusting the end-point. Because length minimality is preserved in the limit, this proves the non-minimality of the original corner. For the spiral $\gamma$, however, this shortening technique fails at the very first step because any blow-up of $\gamma$ at the singular point is a line, that is indeed length minimizing.

\section{Acknowledgments}

It is a pleasure to thank Ben Warhurst and Alessandro Ottazzi for many illuminating discussions on Tanaka prolongation. We also thank Igor Zelenko and Francesco Fassò for some discussions on a preliminary version of the paper.

This work was partially supported by INDAM, University of Padova research project Some analytic and differential geometric aspects in Nonlinear Control Theory, with applications to Mechanics, and Fondazione CaRiPaRo Project Nonlinear Partial Differential Equations: models, analysis, and control-theoretic problems.

\section{References}

[1] A. Agrachev, Any sub-Riemannian metric has points of smoothness, (Russian) Dokl. Akad. Nauk 424 (2009), no. 3, 295-298; translation in Dokl. Math. 79 (2009), no. 1, 45-47.

[2] A. Agrachev, Some open problems, in: Geometric Control Theory and sub-Riemannian Geometry (G. Stefani, U. Boscain, J.-P. Gauthier, 
“"LLMV2'final CAG"” — 2018/8/28 — 9:52 — page 755 — \#33

Extremal polynomials

A. Sary'chev, and M. Sigalotti eds.), Springer, INdAM Series 5 (2014), $1-14$.

[3] A. Agrachev and Y. L. Sachkov, Control Theory from the Geometric Viewpoint, Encyclopaedia of Mathematical Sciences 87, Control Theory and Optimization, II. Springer-Verlag, Berlin, 2004.

[4] E. Gonzales, U. Massari, and I. Tamanini, Boundaries of prescribed mean curvature, Atti Accad. Naz. Lincei Cl. Sci. Fis. Mat. Natur. Rend. Lincei (9) Mat. Appl. 4 (1993), no. 3, 197-206.

[5] M. Grayson and R. Grossman, Models for free nilpotent Lie algebras, J. Algebra 135 (1990), no. 1, 177-191.

[6] E. Hakavuori and E. Le Donne, Non-minimality of corners in subriemannian geometry, Invent. Math. 206 (2016), no. 3, 693-704.

[7] M. Hall Jr., A basis for free Lie rings and higher commutators in free groups, Proc. Amer. Math. Soc. 1 (1950), 575-581.

[8] A. W. Knapp, Lie groups beyond an introduction, Progress in Mathematics 140, Birkhäuser, 2002.

[9] E. Le Donne, G. P. Leonardi, R. Monti, and D. Vittone, Extremal curves in nilpotent Lie groups, Geom. Funct. Anal. 23 (2013), no. 4, 1371-1401.

[10] E. Le Donne, G. P. Leonardi, R. Monti, and D. Vittone, Corners in nonequiregular sub-Riemannian manifolds, ESAIM Control Optim. Calc. Var. 21 (2015), no. 3, 625-634..

[11] E. Le Donne, R. Montgomery, A. Ottazzi, P. Pansu, and D. Vittone, Sard Property for the endpoint map on some Carnot groups, Ann. Inst. H. Poincaré Anal. Non Linéaire 33 (2016), no. 6, 1639-1666.

[12] G. P. Leonardi and R. Monti, End-point equations and regularity of subRiemannian geodesics, Geom. Funct. Anal. 18 (2008), no. 2, 552-582.

[13] R. Montgomery, A Tour of Sub-Riemannian Geometries, Their Geodesics and Applications, AMS, 2002.

[14] R. Monti, Regularity results for sub-Riemannian geodesics, Calc. Var. Partial Differential Equations 49 (2014), no. 1-2, 549-582.

[15] R. Monti, The regularity problem for sub-Riemannian geodesics, Geometric Control Theory and sub-Riemannian Geometry (G. Stefani, U. Boscain, J.-P. Gauthier, A. Sary'chev, and M. Sigalotti eds.), Springer, INdAM Series 5 (2014), 313-332. 
"'LLMV2'final CAG"” — 2018/8/28 — 9:52 — page 756 — \#34

[16] R. Monti, A family of nonminimizing abnormal curves. Ann. Mat. Pura Appl. 193 (2013), no. 6, 1577-1593.

[17] R. Monti, A. Pigati, and D. Vittone, Existence of tangent lines to Carnot-Carathéodory geodesics, Calc. Var. Partial Differential Equations 57 (2018), no. 3, Art. 75, 18 pp.

[18] L. Rifford and E. Trelat, Morse-Sard type results in sub-Riemannian geometry, Math. Ann. 332 (2005), 145-159.

[19] N. Tanaka, On differential systems, graded Lie algebras and pseudogroups, J. Math. Kyoto Univ. 10 (1970), 1-82.

[20] D. Vittone, The regularity problem for sub-Riemannian geodesics, in: Geometric Measure Theory and Real Analysis, 193-226, CRM Series 17, Ed. Norm., Pisa, 2014.

[21] B. Warhurst, Tanaka prolongation of free Lie algebras, Geom. Dedicata 130 (2007), 59-69.

[22] I. Zelenko, Nonregular abnormal extremals of 2-distribution: existence, second variation and rigidity, J. Dynamical and Control Systems 5 (1999), no. 3, 347-383.

[23] I. Zelenko and M. Zhitomirskiū, Rigid paths of generic 2-distributions on 3-manifolds, Duke Math. J. 79 (1995), no. 2, 281-307. 
"'LLMV2'final CAG"” — 2018/8/28 — 9:52 — page 757 — \#35

Extremal polynomials

\section{EnRICo Le Donne}

Department of Mathematics and Statistics

P.O. Box 35, FIN-40014

UNIVERsity of JyVÄSKYLÄ, FinLAND

E-mail address: ledonne@msri.org

Gianpaolo Leonardi

Università di Modena e Reggio Emilia

Dipartimento di Scienze Fisiche, Informatiche e Matematiche

via CAmpi 213/B, 41100, Modena, Italy

E-mail address: gianpaolo.leonardi@unimore.it

Roberto Monti and Davide Vittone

Università di Padova, Dipartimento di Matematica

via Trieste 63, 35121, Padova, Italy

E-mail address: monti@math.unipd.it

E-mail address: vittone@math.unipd.it

Received February 1, 2015 
$\bigoplus$

"'LLMV2 final CAG"” — 2018/8/28 — 9:52 — page 758 — \#36 